\title{
The dam-break problem for Herschel-Bulkley viscoplastic fluids down steep flumes
}

\author{
Christophe Ancey ${ }^{\mathrm{a}, *}$, Steve Cochard ${ }^{\mathrm{b}}$ \\ a School of Architecture, Civil and Environmental Engineering, École Polytechnique Fédérale de Lausanne, 1015 Lausanne, Switzerland \\ ${ }^{\mathrm{b}}$ Department of Mathematics, University of British Columbia, Vancouver V6K 2A5, Canada
}

\section{A R T I C L E I N F O}

\section{Article history:}

Received 2 April 2008

Received in revised form 11 August 2008

Accepted 14 August 2008

\section{Keywords:}

Lubrication theory

Plasticity

Yield stress

Shallow-flow equations

Herschel-Bulkley model

\begin{abstract}
A B S T R A C T
In this paper we investigate the dam-break problem for viscoplastic (Herschel-Bulkley) fluids down a sloping flume: a fixed volume of fluid initially contained in a reservoir is released onto a slope and flows driven by gravitational forces until these forces are unable to overcome the fluid's yield stress. Like in many earlier investigations, we use lubrication theory and matched asymptotic expansions to derive the evolution equation of the flow depth, but with a different scaling for the flow variables, which makes it possible to study the flow behavior on steep slopes. The evolution equation takes on the form of a nonlinear diffusion-convection equation. To leading order, this equation simplifies into a convection equation and reflects the balance between gravitational forces and viscous forces. After presenting analytical and numerical results, we compare theory with experimental data obtained with a long flume. We explore a fairly wide range of flume inclinations from $6^{\circ}$ to $24^{\circ}$, while the initial Bingham number lies in the 0.07-0.26 range. Good agreement is found at the highest slopes, where both the front position and flowdepth profiles are properly described by theory. In contrast, at the lowest slopes, theoretical predictions substantially deviate from experimental data. Discrepancies may arise from the formation of unsheared zones or lateral levees that cause slight flow acceleration.
\end{abstract}

(c) 2008 Elsevier B.V. All rights reserved.

\section{Introduction}

Viscoplastic models are of common use to describe natural gravity-driven flows down steep slopes. Typical examples include mud and debris flows [1-3], snow avalanches [4,5], and lava flows [6]. At first sight, the idea of viscoplastic behavior is very appealing since it explains why natural bulk materials behave like solids when they are at rest and why under some circumstances they yield and start to flow like fluids. Yet, from the rheological point of view, given how difficult it is to characterize the flow properties of natural samples using rheometers, this idea has received little attention so far. From laboratory and outdoor experiments, Dent and Lang [4,7] together with Kern et al. [8] provided evidence that an empirical relation such as the Bingham or Herschel-Bulkley models closely approximates the flow behavior of snow flowing down a flume. The relevance of viscoplasticity to debris flows is still vigorously debated within the scientific community (see Refs. [9-12] and references therein). While small-scale laboratory experiments clearly demonstrated the potential of viscoplastic models to describe the behavior of fine mud and clay dispersions (e.g., kaolin, bentonite)

\footnotetext{
* Corresponding author. Tel.: +41 2169332 87; fax: +41 216936767.

E-mail address: christophe.ancey@epfl.ch (C. Ancey).
}

[3,13-16], large-scale indoor and outdoor experiments carried out with poorly sorted materials have shown a more contrasted and complex behavior: the flow properties depend a great deal on the flow organization, i.e., the existence of lateral levees, a front rich in coarse materials, segregation, as well as entrainment/deposition processes $[9,17,18]$. Field data and comparison with historical events have not settled this controversial issue [19-23] since traces left by debris flows could be interpreted using viscoplastic theory, whereas other clues argue in favor of a Coulomb behavior. The same difficulties arise in the rheology of lava, with an additional degree of complexity induced by temperature and phase changes [6,24-27].

In this delicate context, it is of great interest to gain insight into the dynamic behavior of finite volumes of viscoplastic materials down sloping beds. This issue has attracted growing attention in recent years. Two theoretical approaches have been used to derive the governing equations. In what we can refer to as the Saint-Venant approach, the governing equations are derived by averaging the local mass and momentum balance equations across the stream depth $[3,14,15,28-30]$. The crux is the computation of the bottom shear stress for out-of-equilibrium flows [31,32]. An alternative approach is lubrication theory, which takes its roots in Reynolds' pioneering work. The theory is based on an approximation to the governing equations for shallow slopes and thin low-inertia flows through an asymptotic expansion in the aspect ratio $\varepsilon=H_{*} / L_{*}^{*}$, with 
$H_{*}$ and $L *$ being the flow-depth and length scales, respectively [13,24,33-41]. As pointed out by Balmforth et al. [40], this theory can be extended to steep slopes by changing the scaling that underpins the asymptotic reduction of the local equations.

The objective of this paper is to work out a model describing the behavior of a thin viscoplastic sheet flowing down a sloping bed using lubrication theory. We consider an idealized setting: in a dam-break flow, a fixed volume of a Herschel-Bulkley fluid is instantaneously released and flows down a slope under gravity effects. Contrary to Newtonian fluids [42], the motion is finally arrested when the gravity forces are unable to overcome the resistance force arising from yield stress. In Section 2, we show how the lubrication approximation leads to an evolution equation for the flow depth. In contrast with earlier work, we focus on steep slopes. To leading order $\varepsilon^{0}$, there is no difference between this equation and the one derived using the Saint-Venant approach, but substantial differences arise at higher orders $\varepsilon^{k}(k=1,2 \cdots)$. Theory is then compared with experimental results. Carrying out such experiments is difficult. The reasons are twofold. First, the Herschel-Bulkley model is an idealization of viscoplastic behavior. Most fluids used to date exhibit rheological properties (e.g., viscoelasticity, thixotropy, ageing), which are not accounted for in this model. In fluid rheometry, one is able to explore a narrow range of flow conditions (viscometric flows), which means that the real behavior in more complex flow geometries is unknown to a large degree; in particular, this includes the effect of normal stresses on bulk dynamics and preand post-yielding behaviors. In the 1990s and early 2000s, the typical material used in most experiments was kaolin, a clay suspension which usually exhibits viscoplastic properties. In fact, compared to other clays, kaolin has an unusual behavior, partly because yield stress arises from steric interactions (jamming) between flocs rather than colloidal interactions. In recent years, Carbopol has been increasingly used as a Herschel-Bulkley fluid, but taking a closer look at rheometrical data reveals more subtle behavior, as is usually observed for yield-stress fluids [43]; it should be then kept in mind that the Herschel-Bulkley approximation holds for a finite range of shear rates. Second, tracking the free surface of a timedependent flow remains a challenging task. Here we take advantage of sophisticated image processing techniques [44,45] to measure the flow-depth profiles and front velocity of finite volumes of Carbopol Ultrez 10. In Section 3, we present our experimental data and compare them with theoretical predictions. We then summarize our findings and draw conclusions.

\section{Theory}

\subsection{Setting and scaling}

We consider an infinite plane tilted at an angle $\theta$ to the horizontal. We use a Cartesian coordinate system, where $x$ denotes the downstream coordinate measured from the top of the plane, while $y$ denotes the coordinate normal to the slope (see Fig. 1 ). A rectangular box of length $\ell$, equipped with a gate perpendicular to the slope, and placed at the plane inlet is partially filled with a volume $V$ of a Herschel-Bulkley fluid with density $\rho$. The rear end of this reservoir is chosen to be the origin of the $x$-axis. At time $t=0$, the lock gate is suddenly opened and the fluid is released onto the plane. Initially the flow depth is denoted by

$h_{i}(x)=h_{\mathrm{g}}+(x-\ell) \tan \theta$,

with $h_{\mathrm{g}}$ the gate aperture, for $0 \leq x \leq \ell$ and $h_{i}=0$ for $x>\ell$. We are interested in determining the flow-depth profile $h(x, t)$ and the position $x_{\mathrm{f}}$ of the front, i.e., the point where the flow depth drops to zero: $h\left(x_{\mathrm{f}}\right)=0 . h$ is the flow depth measured normal to the plane.

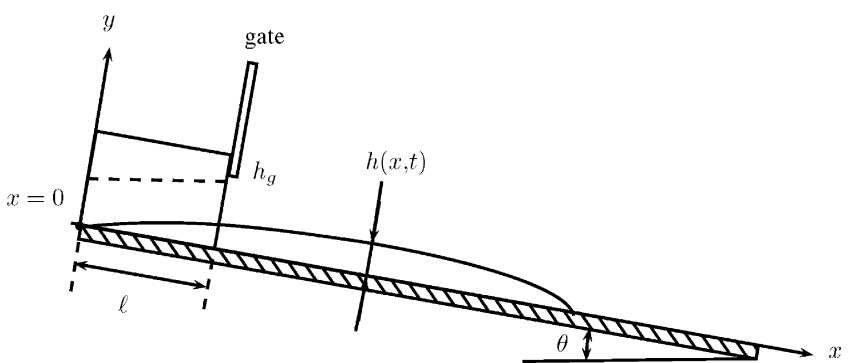

Fig. 1. Sketch defining the flow configuration.

Conservation of mass and momentum read

$\frac{\partial u}{\partial x}+\frac{\partial v}{\partial y}=0$

$\rho \frac{\mathrm{d} u}{\mathrm{~d} t}=\rho g \sin \theta-\frac{\partial p}{\partial x}+\frac{\partial \sigma_{x x}}{\partial x}+\frac{\partial \sigma_{x y}}{\partial y}$,

$\rho \frac{\mathrm{d} v}{\mathrm{~d} t}=-\rho g \cos \theta-\frac{\partial p}{\partial y}+\frac{\partial \sigma_{x y}}{\partial x}+\frac{\partial \sigma_{y y}}{\partial y}$,

where $g$ denotes gravity acceleration, $\sigma_{x x}, \sigma_{y y}$, and $\sigma_{x y}$ are the normal stress in the $x$-direction, normal stress in the $y$-direction, and shear stress, respectively. They are the components of the extrastress tensor [12]

$\sigma=\left(\frac{\tau_{\mathrm{c}}}{\dot{\gamma}}+2^{n} K \dot{\gamma}^{n-1}\right) \mathbf{d}, \quad$ for $\tau>\tau_{\mathrm{c}}$,

$\mathbf{d}=0$ for $\tau \leq \tau_{\mathrm{c}}$,

where $\mathbf{d}$ is the strain-rate tensor, $\dot{\gamma}=\sqrt{\frac{1}{2} \operatorname{tr}(\mathbf{d} \cdot \mathbf{d})}$ is the second invariant of $\mathbf{d}$, and $\tau=\sqrt{\frac{1}{2} \operatorname{tr}(\sigma \cdot \sigma)}$ is the second invariant of the extra-stress tensor $\sigma$. The relation $\tau=\tau_{\mathrm{c}}$ is referred to as the yield condition. In this constitutive equation, $n$ is an index usually satisfying $n \leq 1, K$ is the consistency, and $\tau_{\mathrm{c}}$ is the yield stress. The Eqs. (2)-(4) are subject to the kinematic boundary conditions

$u=v=0$ for $y=0$

at the bottom, while at the free surface (which is assumed to be stress free), we have

$(-p 1+\sigma) \cdot \mathbf{n}=0$ for $y=h$,

with $\mathbf{n}=\left(-\partial_{x} h, 1\right)$ a vector normal to the free surface, together with the kinematic condition

$v=\frac{\partial h}{\partial t}+u \frac{\partial h}{\partial x}$ for $y=h$.

Mass conservation also implies that

$\int_{0}^{x_{\mathrm{f}}} h(x, t) \mathrm{d} x=V=\frac{1}{2} \ell\left(2 h_{\mathrm{g}}-\ell \tan \theta\right)$.

We introduce the aspect ratio

$\varepsilon=\frac{H_{*}}{L_{*}}$,

which is considered to be low. A natural choice to define the typical scales introduced in this ratio is to take the reservoir dimensions: $H_{*}=h_{\mathrm{g}}$ and $L_{*}=\ell$, but this produces artificially high $\varepsilon$ values in the early stages of the release. Another choice is to consider that the final state provides an appropriate crossstream length scale: $H_{*}=h_{\mathrm{c}}=\tau_{\mathrm{c}} /(\rho g \sin \theta)$; volume conservation then implies $L_{*}=\ell_{\mathrm{C}}=V / H_{*}$. 
We introduce the generalized Reynolds and Bingham numbers

$R e=\rho \frac{U_{*} H_{*}}{K\left(U_{*} / H_{*}\right)^{n-1}}$ and $B i=\frac{\tau_{\mathrm{c}}}{K\left(U_{*} / H_{*}\right)^{n}}$.

As usual, the Reynolds number can be interpreted as the ratio of inertia to viscous forces, while the Bingham number is a dimensionless yield stress (relative to the viscous forces); the Bingham number is sometimes referred to as the Herschel-Bulkley or Oldroyd number.

We use the following dimensionless variables

$x=L_{*} \tilde{x}, \quad y=\varepsilon L_{*} \tilde{y}, \quad$ and $t=T_{*} \tilde{t}$,

$u=U_{*} \tilde{u}$ and $v=\varepsilon U_{*} \tilde{v}$,

$\sigma_{x x}=K\left(\frac{U_{*}}{H_{*}}\right)^{n} \tilde{\sigma}_{\tilde{x} \tilde{x}}$ and $\sigma_{y y}=K\left(\frac{U_{*}}{H_{*}}\right)^{n} \tilde{\sigma}_{\tilde{y} \tilde{y}}$,

$\sigma_{x y}=K\left(\frac{U_{*}}{H_{*}}\right)^{n} \tilde{\sigma}_{\tilde{x} \tilde{y}}$ and $p=P_{*} \tilde{p}$

with

$P_{*}=\rho g H_{*} \cos \theta$ and $T_{*}=\frac{L_{*}}{U_{*}}$,

the pressure and time scales, respectively. The velocity scale $U_{*}$ depends on the flow regime considered. Hereafter we will address two limiting flow regimes. We refer to the diffusive regime as the flow for which the pressure gradient is counterbalanced by viscous forces (acting in the cross-stream direction) and bed inclination is shallow. This gives the velocity scale $U_{*}=$ $U_{\text {diff }}=(\rho g \cos \theta / K)^{1 / n} H_{*}^{1+2 / n} / L_{*}^{1 / n}$ and imposes the constraint $\tan \theta / \varepsilon=\mathrm{O}(1)$; see Appendix A. The other regime is referred to as the slope-dominated regime. It corresponds to the limiting flow conditions where the pressure gradient (in the downstream direction) becomes negligible compared to the gravity and viscous forces (see Section 2.2); the flow reaches a near-equilibrium regime, where viscous forces balance gravity acceleration. The velocity scale is then

$U_{*}=U_{\text {conv }}=(\rho g \sin \theta / K)^{1 / n} H_{*}{ }^{1+(1 / n)}$.

Note that with this scaling and because of mass conservation, the dimensionless stress and strain-rate invariants are

$\tilde{\tau}=\left|\tilde{\sigma}_{\tilde{x} \tilde{y}}+\tilde{\sigma}_{\tilde{x} \tilde{x}}\right|$ and $\tilde{\gamma}=\frac{1}{2}\left[4\left(\varepsilon \partial_{\tilde{x}} \tilde{u}\right)^{2}+\left(\partial_{\tilde{y}} \tilde{u}+\varepsilon^{2} \partial_{\tilde{x}} \tilde{v}\right)^{2}\right]^{1 / 2}$.

The yield condition in a dimensionless form is then $\tilde{\tau}=B i$.

\subsection{Slope-dominated regime}

The scaled governing equations are made up of the mass and momentum balance equations:

$\frac{\partial u}{\partial x}+\frac{\partial v}{\partial y}=0$

$\varepsilon R e \frac{\mathrm{d} u}{\mathrm{~d} t}=1-\varepsilon \cot \theta \frac{\partial p}{\partial x}+\varepsilon \frac{\partial \sigma_{x x}}{\partial x}+\frac{\partial \sigma_{x y}}{\partial y}$,

$\varepsilon^{2} R e \frac{\mathrm{d} v}{\mathrm{~d} t}=-\cot \theta\left(1+\frac{\partial p}{\partial y}\right)+\varepsilon \frac{\partial \sigma_{x y}}{\partial x}+\frac{\partial \sigma_{y y}}{\partial y}$,

where the tilde decoration has been dropped. The stress boundary conditions ( 8 ) at the free surface $y=h(x, t)$ become

$\sigma_{x y}=\varepsilon \frac{\partial h}{\partial x}\left(\sigma_{x x}-p \cot \theta\right)$

$\varepsilon \frac{\partial h}{\partial x} \sigma_{x y}=\sigma_{y y}-p \cot \theta$ while the kinematic boundary conditions are $v=\mathrm{d} h / \mathrm{d} t$ for $y=h(x, t)$ and $u=v=0$ for $y=0$. The flow depth vanishes at the front:

$h\left(x_{f}, t\right)=0$.

Mass conservation also implies that the volume of the flow is preserved:

$\int_{0}^{x_{\mathrm{f}}} h(x, t) \mathrm{d} x=V$.

The initial value for $h$ is

$h(x, 0)=h_{\mathrm{g}}+\kappa(x-\ell)$,

with $\kappa=L * \tan \theta / H_{*}=\tan \theta / \varepsilon$.

We use perturbation methods and matched asymptotic expansions to study the behavior of the viscoplastic fluid released down an inclined plane [46]. There are two issues that must be addressed:

- The first issue is related to the dynamics of the flow and is somehow independent of the constitutive equation: as sketched in Fig. 1, the flow can be split into two different regions: the body and the front, where the flow depth drops to zero. For the body, the leading-order terms of the governing equations are obtained by removing the contributions that depend on $\varepsilon$ in Eqs. (19) and (20). As readily seen in the momentum equations, the bulk of the flow is in a nearly steady regime, where gravity acceleration is counterbalanced by the cross-stream gradient of the shear stress. Since this behavior conflicts with the boundary condition (23), a boundary-layer correction is needed at the front. Indeed, the steady-regime solution is no longer valid within the tip region because the pressure gradient $\varepsilon \partial_{x} p$ becomes non-negligible. The dynamics of the front is then controlled by the balance between the streamwise pressure and stress gradients, $\varepsilon \partial_{x} p \sim \varepsilon h / \xi$ and $\partial_{y} \sigma_{x y} \sim(u / h)^{n} / h$, respectively:

$\varepsilon \frac{h}{\xi} \sim \frac{(u / h)^{n}}{h}$,

with $\xi=x-x_{\mathrm{f}}$ and $u \propto h^{1 /(n+1)}$. The extent of the boundary layer can then be estimated as $\xi=\mathrm{O}(\varepsilon h)$. In this subsection, we will describe the solution for the body, referred to as the outer solution, while in the next subsection, attention will be focused on the boundary-layer correction (called the inner solution). The inner solution smoothly connects to the outer solution at $x=x_{\mathrm{f}}$.

- The second issue arises from the occurrence of a nearly steady regime while the fluid is viscoplastic. In a genuinely steady uniform regime, part of the fluid is sheared close to the bottom boundary while there is a rigid plug flow near the free surface [47]. Since to leading order, the governing equations are similar to those describing the steady uniform regime, it is expected that the plug structure subsists here, but it cannot be a true plug because this would conflict with the flow structure (which depends on $x$ ). To avoid inconsistencies in the perturbation analysis, we follow the treatment suggested by Balmforth and Craster [38], which consists in considering two asymptotic expansions (one for the sheared layer and the other one for the pseudo-plug layer) and matching them through a 'fake' yield surface, i.e., the interface at which the second stress invariant $\tau$ is at the yield-stress value to leading order. We refer the reader to Ref. [38] for further developments; here we will focus on the leading-order terms and skip details in the matching of solutions at the fake yield surface.

Anticipating the existence of a pseudo-plug [38], where the strain-rate invariant $\dot{\gamma}$ is virtually zero, we distinguish 
- a sheared layer close to the bottom $(0 \leq y \leq Y)$; and

- a plug zone near the free surface $(Y \leq y \leq h)$.

$Y$ denotes the position of the interface ('fake' yield surface) between the sheared and pseudo-plug layers and is unknown for the moment. In the shear layer, the fluid is fully sheared; with the scaling (11)-(15), we have $\sigma_{x x}=\mathrm{O}(\varepsilon)$ and $\sigma_{y y}=\mathrm{O}(\varepsilon)$, but $\sigma_{x y}=\mathrm{O}(1)$. We then introduce the $\varepsilon$-expansions: $\sigma_{y y}=\varepsilon \sigma_{1, y y}+\ldots$, $\sigma_{x x}=\varepsilon \sigma_{1, x x}+\ldots$, and $\sigma_{x y}=\sigma_{0, x y}+\varepsilon \sigma_{1, x y}+\ldots$. We pose the expansion $u(x, y, t)=u_{0}(x, y, t)+\varepsilon u_{1}(x, y, t)+\cdots$, leading to $\dot{\gamma}=\frac{1}{2}\left|\partial_{y} u_{0}\right|+$ $\frac{1}{2} \varepsilon \partial_{y} u_{1}+\mathrm{O}\left(\varepsilon^{2}\right)$.

Within the plug layer, there is little deformation, $\dot{\gamma}$ being close to zero. In that case, we pose $u(x, y, t)=$ $u_{0}^{\prime}(x, t)+\varepsilon u_{1}^{\prime}(x, y, t)+\cdots$, where the dependence on $y$ in the zero-order term has disappeared. Because of this, we must expand the stress components differently when $n<1$ : $\sigma_{y y}=\sigma_{0, y y}+\varepsilon^{n} \sigma_{n, y y}+\varepsilon^{1} \sigma_{1, y y}+\varepsilon^{n+1} \sigma_{n+1, y y}, \quad \sigma_{x x}=\sigma_{0, x x}+\varepsilon^{n} \sigma_{n, x x}+\ldots$, $\sigma_{x y}=\sigma_{0, x y}+\varepsilon \sigma_{1, x y}+\ldots$, and $p=\varepsilon^{n-1} p_{n-1}+p_{0}+\varepsilon p_{1}+\ldots$. Note that for Bingham fluids $(n=1)$, the expansions are regular power series of $\varepsilon$. Since $u_{0}^{\prime}$ does not depend on $y$, we have $\dot{\gamma}=\frac{1}{2} \varepsilon \sqrt{\left(\partial_{y} u_{1}^{\prime}\right)^{2}+4\left(\partial_{x} u_{0}^{\prime}\right)^{2}}+\mathrm{O}\left(\varepsilon^{2}\right)$, showing that $\dot{\gamma}$ is order $\varepsilon$ unless $\partial_{y} u_{1}^{\prime}$ and $\partial_{x} u_{0}^{\prime}$ vanish simultaneously.

We also introduce $Y=Y_{0}+\varepsilon Y_{1}+\ldots, \quad h=h_{0}+\varepsilon h_{1}+\ldots$, and $\tau=\tau_{0}+\varepsilon \tau_{1}+\ldots$. To order $\varepsilon^{0}$, we have to solve

$0=1+\frac{\partial \sigma_{0, x y}}{\partial y}$,

$0=-1-\frac{\partial p_{0}}{\partial y}$ for $y \leq Y_{0}$

$0=-\cot \theta+\frac{\partial}{\partial y}\left(\sigma_{0, y y}-p_{0} \cot \theta\right)$ for $y \geq Y_{0}$,

subject to

$\sigma_{0, y y}-p_{0} \cot \theta=0$ and $\sigma_{0, x y}=0$ for $y=h_{0}$.

In the limit of $R e \rightarrow 0$ and to order $\varepsilon$ in (19) and (20), we obtain

$0=-\cot \theta \frac{\partial p_{0}}{\partial x}+\frac{\partial \sigma_{1, x y}}{\partial y}$,

$0=-\cot \theta \frac{\partial p_{1}}{\partial y}+\frac{\partial \sigma_{0, x y}}{\partial x}+\frac{\partial \sigma_{1, y y}}{\partial y}$,

subject to

$\left\{\begin{array}{l}\sigma_{1, x y}=-h_{1} \frac{\partial \sigma_{0, x y}}{\partial y}-\cot \theta \frac{\partial h_{0}}{\partial x} p_{0} \\ \sigma_{1, y y}-p_{1} \cot \theta=\cot \theta \frac{\partial p_{0}}{\partial y} h_{1}+\frac{\partial h_{0}}{\partial x} \sigma_{0, x y}\end{array}\right.$ at $y=h_{0}$.

Solving Eqs. (26)-(29) leads to the following stress fields

$\sigma_{0, x y}=h_{0}-y$,

$\sigma_{0, y y}-p_{0} \cot \theta=\left(h_{0}-y\right) \cot \theta$,

which are identical to the expressions found for a steady uniform flow. The yield condition is $\tau_{0}=\left|\sigma_{0, x y}\right|=B i$, from which we deduce that the position of the fake yield surface is given by $Y_{0}=h_{0}-B i$.

To order $\varepsilon$, we deduce from (30)-(32)

$\sigma_{1, x y}=h_{1}-\cot \theta \frac{\partial h_{0}}{\partial x}\left(h_{0}-y\right)$,

$\sigma_{1, y y}-p_{1} \cot \theta=-h_{1} \cot \theta+\frac{\partial h_{0}}{\partial x}\left(h_{0}-y\right)$.
We now pursue by inferring the velocity field from the stresses. Within the shear layer, the constitutive equation to orders $\varepsilon^{0}$ and $\varepsilon^{1}$ are

$\sigma_{0, x y}=B i+\left(\frac{\partial u_{0}}{\partial y}\right)^{n}$,

$\sigma_{1, x y}=n\left(\frac{\partial u_{0}}{\partial y}\right)^{n-1} \frac{\partial u_{1}}{\partial y}$,

while in the pseudo-plug layer, it takes on the form

$\sigma_{0, x y}=\frac{B i}{\sqrt{4\left(\partial_{x} u_{0}^{\prime}\right)^{2}+\left(\partial_{y} u_{1}^{\prime}\right)^{2}}} \frac{\partial u_{1}^{\prime}}{\partial y}$.

Using velocity continuity at the interface $y=Y_{0}$, we obtain the cross-stream velocity to order $\varepsilon^{0}$

$u_{0}(x, y, t)=\frac{n\left(Y_{0}^{1+(1 / n)}-\left(Y_{0}-y\right)^{1+(1 / n)}\right)}{n+1}$ for $y \leq Y_{0}$,

$u_{0}^{\prime}(x, t)=\frac{n}{n+1} Y_{0}^{1+(1 / n)}$ for $y \geq Y_{0}$,

together with its correction to order $\varepsilon^{1}$

$u_{1}(x, y, t)=f(x, y, t)-f(x, 0, t)$ for $y \leq Y_{0}$,

$u_{1}^{\prime}(x, y, t)=2 Y_{0}^{1 / n} \partial_{x} h_{0} \sqrt{\left(y-Y_{0}\right)\left(2 h_{0}-Y_{0}-y\right)}$ for $y \geq Y_{0}$,

with $f=\left(Y_{0}-y\right)^{1 / n}\left[\cos \theta\left(n \mathrm{Bi}+h_{0}-y\right) \partial_{x} h_{0}-(n+1) h_{1}\right] /(n+1)$. Fig. 2 shows a typical velocity profile at leading order together with its first-order correction. $\varepsilon$ was set to 0.1 and two values were considered for the streamwise gradient of the flow depth: $\partial_{x} h_{0}=0.1$ (dashed line) and $\partial_{x} h_{0}=0.01$ (dotted line). The flow-depth gradient has strong influence on the shape of the velocity profile. Note also that since the shear-layer and plug solutions were patched together at the yield surface $y=Y_{0}$, an unrealistic kink at $y=Y_{0}$ arises in the first-order velocity profile, as seen for $\partial_{x} h_{0}=0.1$. In fact, the two solutions should have been connected over a region of width $\varepsilon$ centered around the yield surface because the shear-stress expansion is non-uniform when $\partial_{x} u_{0}$ is order $\varepsilon$ (e.g., see Appendix A in Ref. [38]).

The integration of the cross-stream velocity profiles provides the flow-depth averaged velocity

$\bar{u}_{0}=\frac{n}{(n+1)(2 n+1)} \frac{Y_{0}^{1+(1 / n)}\left(h_{0}+\left(B i+h_{0}\right) n\right)}{h_{0}}$.

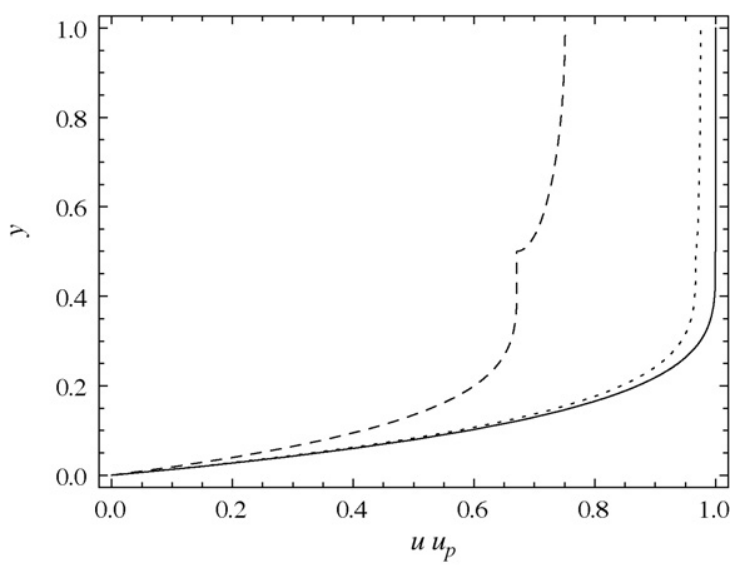

Fig. 2. Normalized velocity profile: velocity profile at order $\varepsilon^{0}$ (solid line) and firstorder correction (dashed or dotted line). The first-order correction was computed for $\partial_{x} h_{0}=0.1$ (dashed line) and $\partial_{x} h_{0}=0.01$ (dotted line). Computations made for $\theta=12^{\circ}$, $B i=0.5, n=1 / 3, \varepsilon=0.1$, and $h_{1}=0 . u_{\mathrm{p}}$ is the plug velocity. 
We finally obtain an evolution equation for $h$ in the form of a nonlinear convection equation.

$\frac{\partial h}{\partial t}+\frac{\partial}{\partial x} F(h)=0$,

with $Y=\max (h-B i, 0)$ and

$F(h)=n Y^{1+1 / n} \frac{(2 n+1) h-n Y}{(2 n+1)(n+1)}$.

Eq. (45) has also been obtained by a number of authors, including Balmforth et al. [40] and Huang and García [14]. These latter authors used a Saint-Venant approach to derive the equations of motion of a viscoplastic sheet flowing down a sloping bed. Using singular perturbations techniques, they found that the outer solution (i.e., the solution representing the flow behavior far from the front) was given by Eq. (45); behavior close to the front was described by seeking the inner solution.

Eq. (45) is a nonlinear convection equation, which can be solved analytically using the method of characteristics. This technique has been used in a number of related problems [14,28,48,49], usually with the additional assumption of a point source as initial condition. In that particular case, the solution to the nonlinear evolution Eq. (45) is a similarity solution. In Appendix B, we solve the full initial-boundary-value problem (45) subject to (23)-(25) using the method of characteristics. The main difference with the treatment used by Huang and García [14,28] lies in the occurrence of two waves, originally emanating from each end of the volume released (shock and rarefaction waves), which then collapse to form a single wave. This analytical solution will be used in the sequel to plot the outer solution in Figs. 3-12.

\subsection{Behavior within the tip region}

As shown in the previous subsection, there is a boundary layer of size $\varepsilon$ at the front. To see what is occurring in this boundary layer, we make the following change of variable

$x^{\prime}=\frac{x-x_{\mathrm{f}}(t)}{\varepsilon}$.

In the mobile frame attached to the front, the dominant balance in the momentum balance Eq. (19) is between the streamwise gradient of the pressure and the cross-stream gradient of the shear stress, suggesting that the proper velocity scale is now $U_{\text {diff }}=\mathrm{O}\left(\varepsilon^{1 / n} U_{*}\right)\left(U_{*}=U_{\text {conv }}\right)$ like for the diffusive regime. The flow depth must then scale as $h=\mathrm{O}\left(\varepsilon^{1 /(n+1)}\right)$ so that the streamwise gradient of the pressure balances the cross-stream gradient of the shear stress provided that
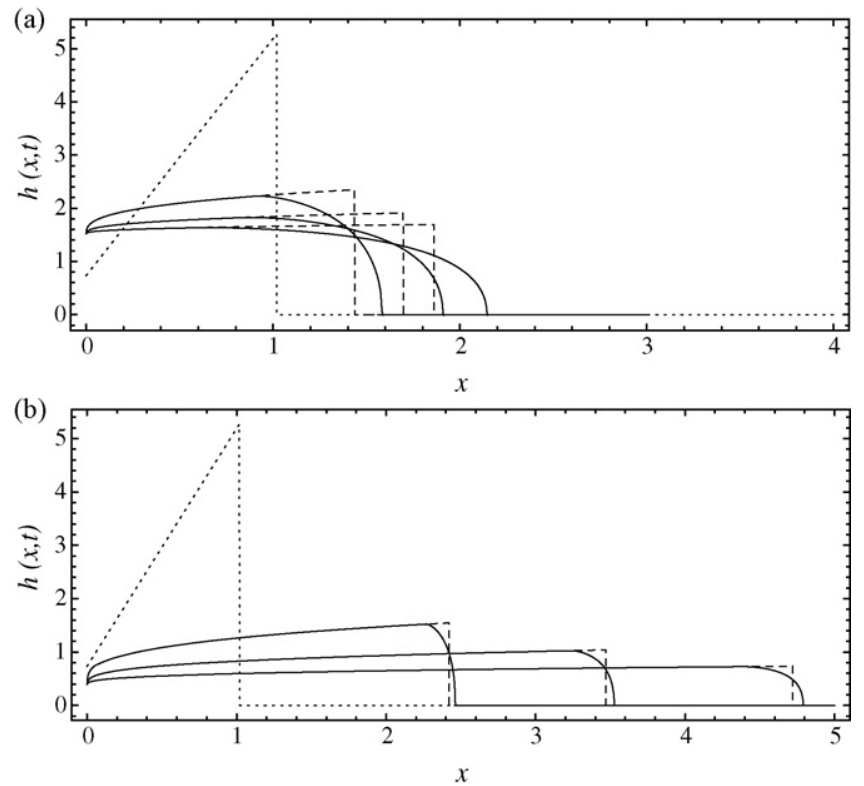

Fig. 3. Flow-depth profiles at times $t=0$ (dotted line), $t=10, t=100$, and $t=1000$ (solid lines) obtained by taking the composite solution. The dashed lines stand for the outer solution at the same times. (a) $\theta=6^{\circ}, B i=1.52, \varepsilon=0.1, \kappa=1.05, n=0.388$ (values drawn for run (a) in Table 5). (b) $\theta=24^{\circ}, B i=0.36, \varepsilon=0.1, \kappa=0.45, n=0.388$ (values drawn for run (a) in Table 2).

$\mathrm{O}\left(\cot \theta \varepsilon^{1 /(n+1)}\right)=1$; note that a similar constraint is met for the diffusive regime. For a fully sheared material, this scaling suggests that $\sigma_{x y} \sim\left(\partial_{y} u\right)^{n} \sim\left(\varepsilon^{1 / n-1 /(n+1)} u^{\prime} / h^{\prime}\right)^{n}=\mathrm{O}\left(\varepsilon^{1 /(n+1)}\right)$ while $\sigma_{x x} \sim\left(\partial_{y} u\right)^{n-1} \varepsilon \partial_{x} u \sim \varepsilon^{2 /(n+1)}\left(u^{\prime} / h^{\prime}\right)^{n}=\mathrm{O}\left(\varepsilon^{2 /(n+1)}\right)$. We now embody this scaling analysis into an asymptotic analysis by substituting the following stretched variables into the governing Eqs. (18)-(24): $\quad x=x_{\mathrm{f}}+\varepsilon x^{\prime}, \quad y=\varepsilon^{1 /(n+1)} y^{\prime}, \quad t=\varepsilon t^{\prime}, \quad u=\varepsilon^{1 / n} u_{0^{\prime}}+\ldots$, $h=\varepsilon^{1 /(n+1)} h_{0^{\prime}}+\cdots, \sigma_{x x}=\varepsilon^{2 /(n+1)} \sigma_{0, x^{\prime} x^{\prime}}+\cdots, \sigma_{y y}=\varepsilon^{2 /(n+1)} \sigma_{0, y^{\prime} y^{\prime}}^{\prime}+\cdots$, $\sigma_{x y}=\varepsilon^{1 /(n+1)} \sigma_{0, x^{\prime} y^{\prime}}^{\prime}+\cdots$, and $p=\varepsilon^{1 /(n+1)} p_{0^{\prime}}+\cdots$.

The re-scaled momentum balance equations are

$$
\begin{aligned}
\operatorname{Re}\left(\frac{\mathrm{d} u}{\mathrm{~d} t^{\prime}}-\dot{x}_{\mathrm{f}} \frac{\partial u}{\partial x^{\prime}}\right)=1 & -\cot \theta \frac{\partial p}{\partial x^{\prime}}+\frac{\partial \sigma_{x^{\prime} x^{\prime}}}{\partial x^{\prime}}+\frac{1}{\varepsilon^{1 /(n+1)}} \frac{\partial \sigma_{x^{\prime} y^{\prime}}}{\partial y^{\prime}} \\
\varepsilon R e\left(\frac{\mathrm{d} v}{\mathrm{~d} t^{\prime}}-\dot{x}_{\mathrm{f}} \frac{\partial v}{\partial x^{\prime}}\right)= & -\cot \theta\left(1+\frac{1}{\varepsilon^{1 /(n+1)}} \frac{\partial p}{\partial y^{\prime}}\right)+\frac{\partial \sigma_{x^{\prime} y^{\prime}}}{\partial x^{\prime}} \\
& +\frac{1}{\varepsilon^{1 /(n+1)}} \frac{\partial \sigma_{y^{\prime} y^{\prime}}}{\partial y^{\prime}}
\end{aligned}
$$
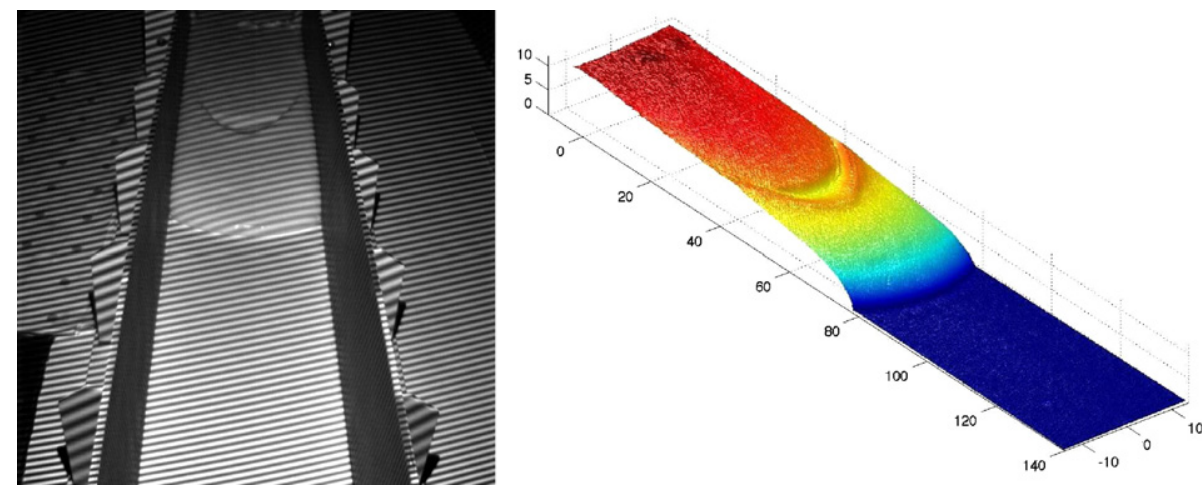

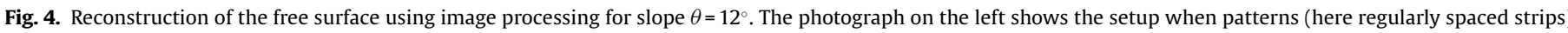
are projected. The picture on the right shows the reconstructed free surface. Figure drawn from Ref. [44]. 

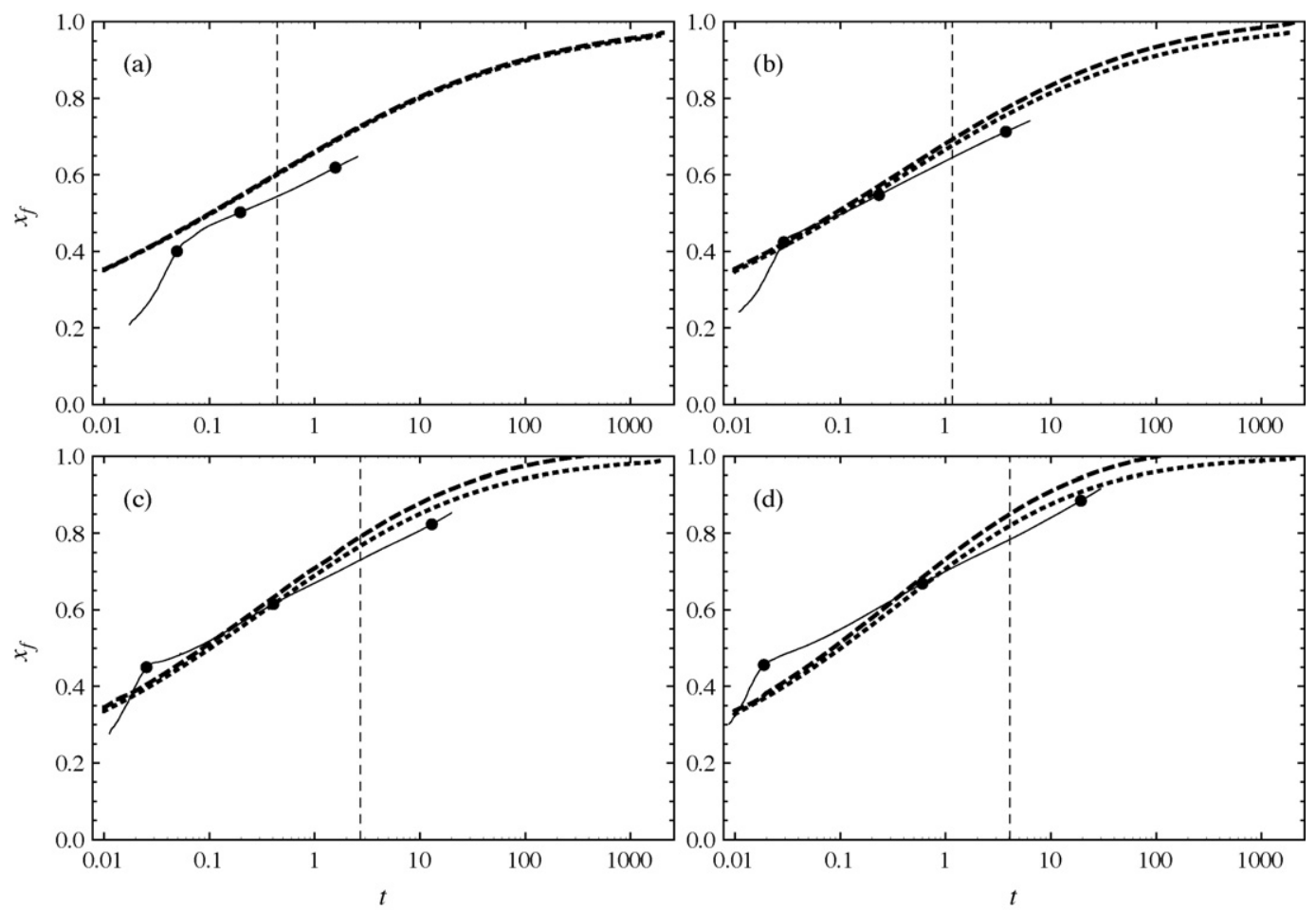

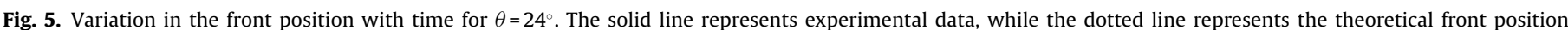

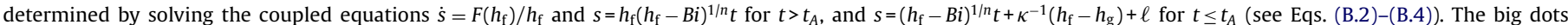

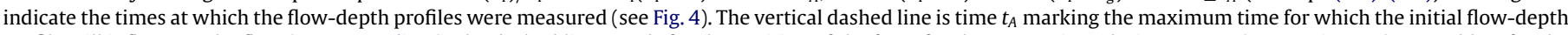

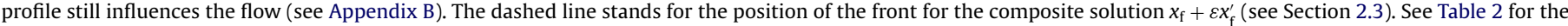
flow conditions pertaining to runs (a) through (d).

The stress boundary conditions at the free surface $y=h(x, t)$ are

$\sigma_{x y}=\frac{\partial h}{\partial x^{\prime}}\left(\sigma_{x x}-p \cot \theta\right)$,

$\frac{\partial h}{\partial x^{\prime}} \sigma_{x y}=\sigma_{y y}-p \cot \theta$.

The matching conditions also demand that the stress fields smoothly connect to the outer solution for $x^{\prime} \rightarrow-\infty$; among others,
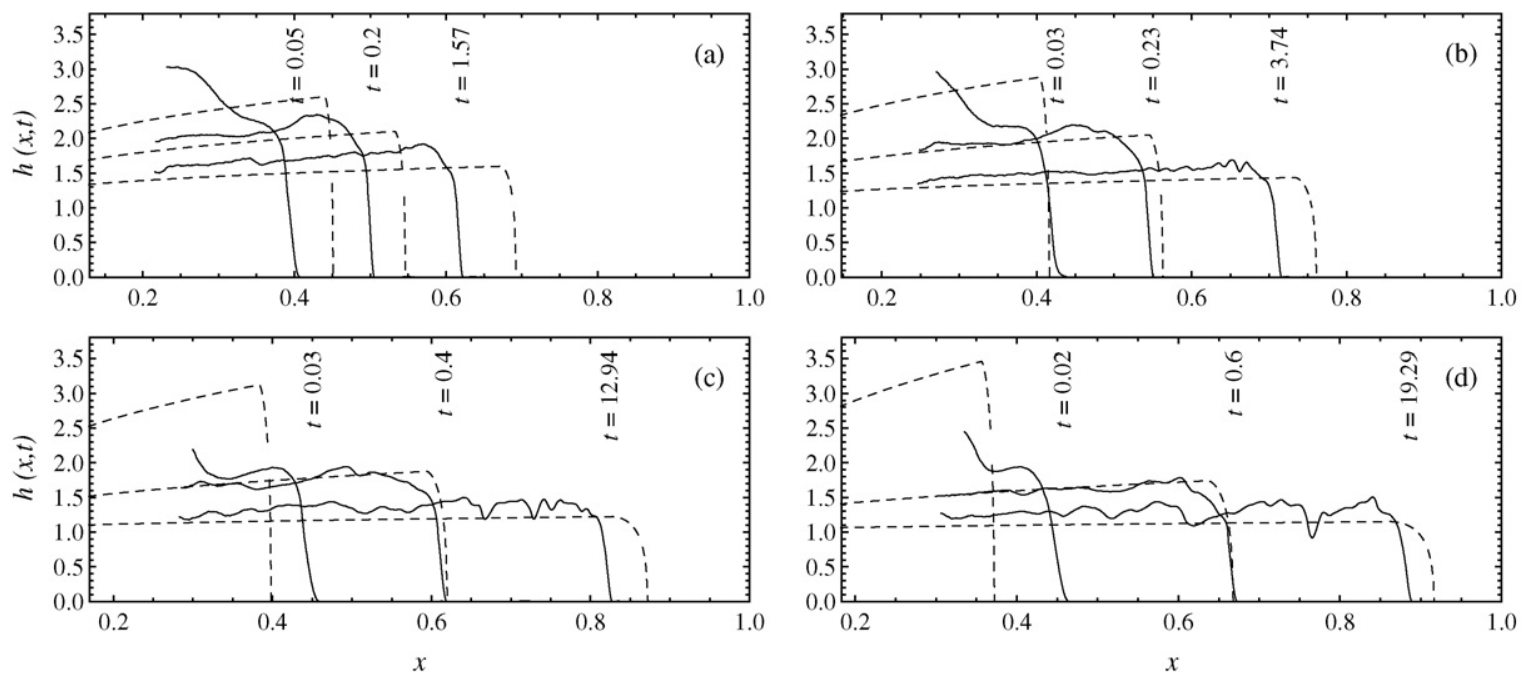

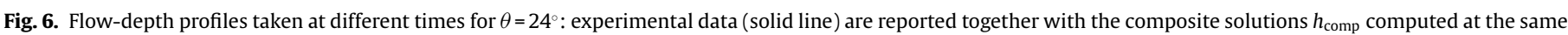
times (dashed line). The dimensionless time at which the profile is taken is also indicated just above the front; these times correspond to the dots plotted in Fig. 3 . 

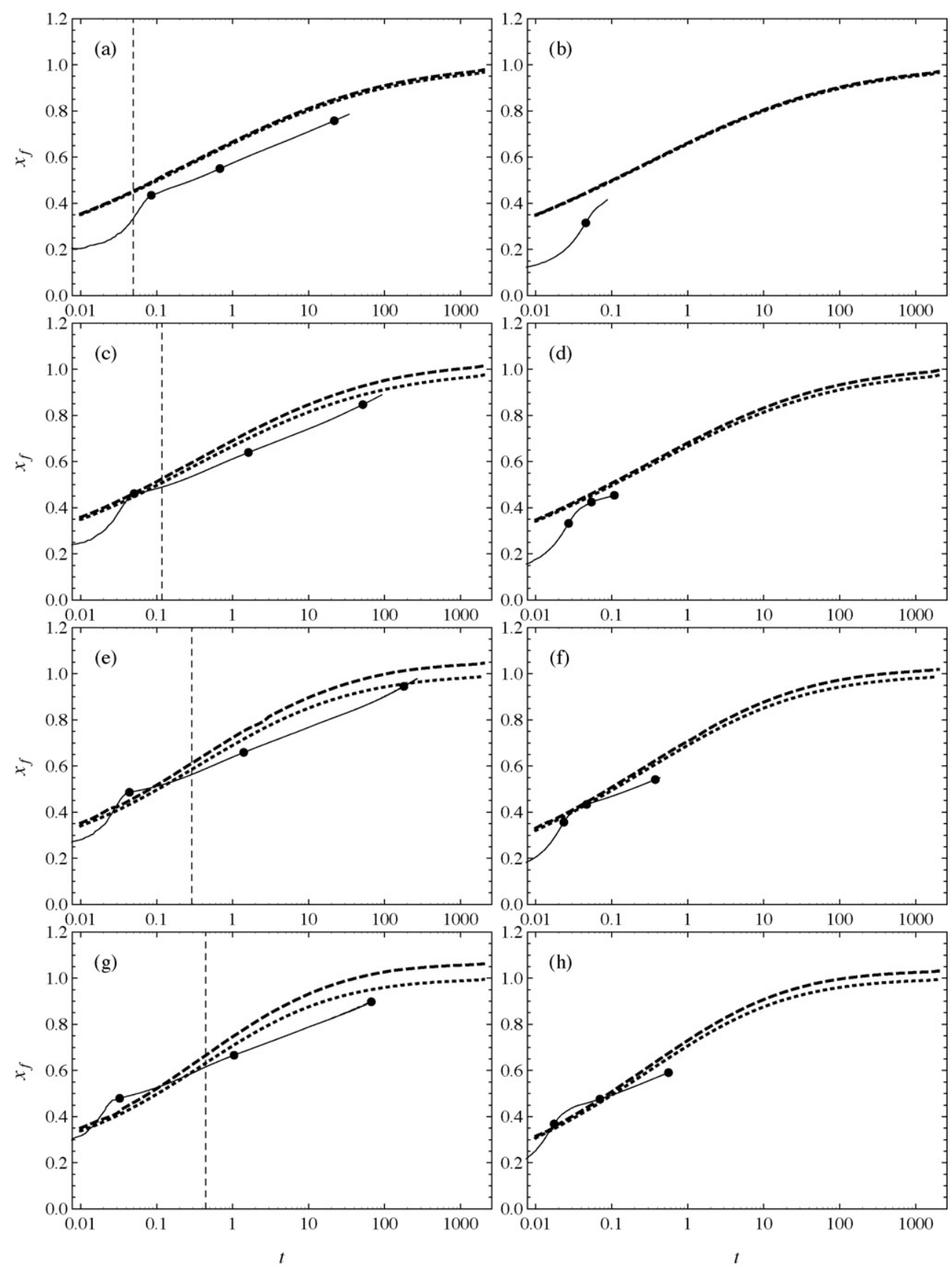

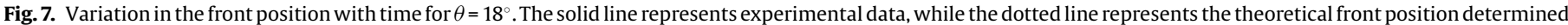

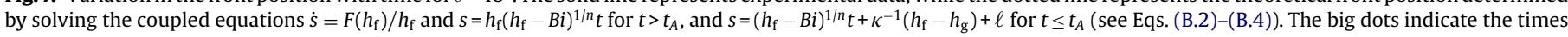

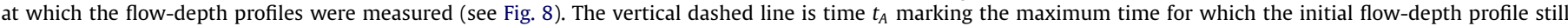
influences the flow (see Appendix B). The dashed line stands for the position of the front for the composite solution $x_{\mathrm{f}}+\varepsilon x_{\mathrm{f}}^{\prime}($ see Section 2.3 ).

With the latter expression, we can derive the velocity profile (see Appendix A):

$u_{0}=\frac{n}{n+1}\left(1-\cot \theta \partial_{\chi^{\prime}} h_{0}\right)\left\{\begin{array}{l}Z_{0}^{1+(1 / n)}-\left(Z_{0}-y\right)^{1+(1 / n)} \\ z_{0}^{1+(1 / n)}\end{array}\right.$,

where

$$
Z_{0}=\max \left(h-\frac{B i}{\left|1-\cot \theta \partial_{\chi^{\prime}} h_{0}\right|}\right)
$$

Integrating this profile leads to the flow-depth averaged velocity and then the evolution equation for the flow depth

$\frac{\partial h_{0}}{\partial t^{\prime}}+\frac{\partial}{\partial x^{\prime}} G\left(h_{0}\right)=0$

$G\left(h_{0}\right)=n Z_{0}^{1+(1 / n)} \frac{(2 n+1) h_{0}-n Z_{0}}{(2 n+1)(n+1)}\left(1-\cot \theta \frac{\partial h_{0}}{\partial x^{\prime}}\right)^{1 / n}$,

and subject to the boundary condition $\lim _{x^{\prime} \rightarrow-\infty}$. Since the volume of fluid contained in the inner region is order $\varepsilon$, mass is merely redistributed with no creation or loss within the head. The initial 

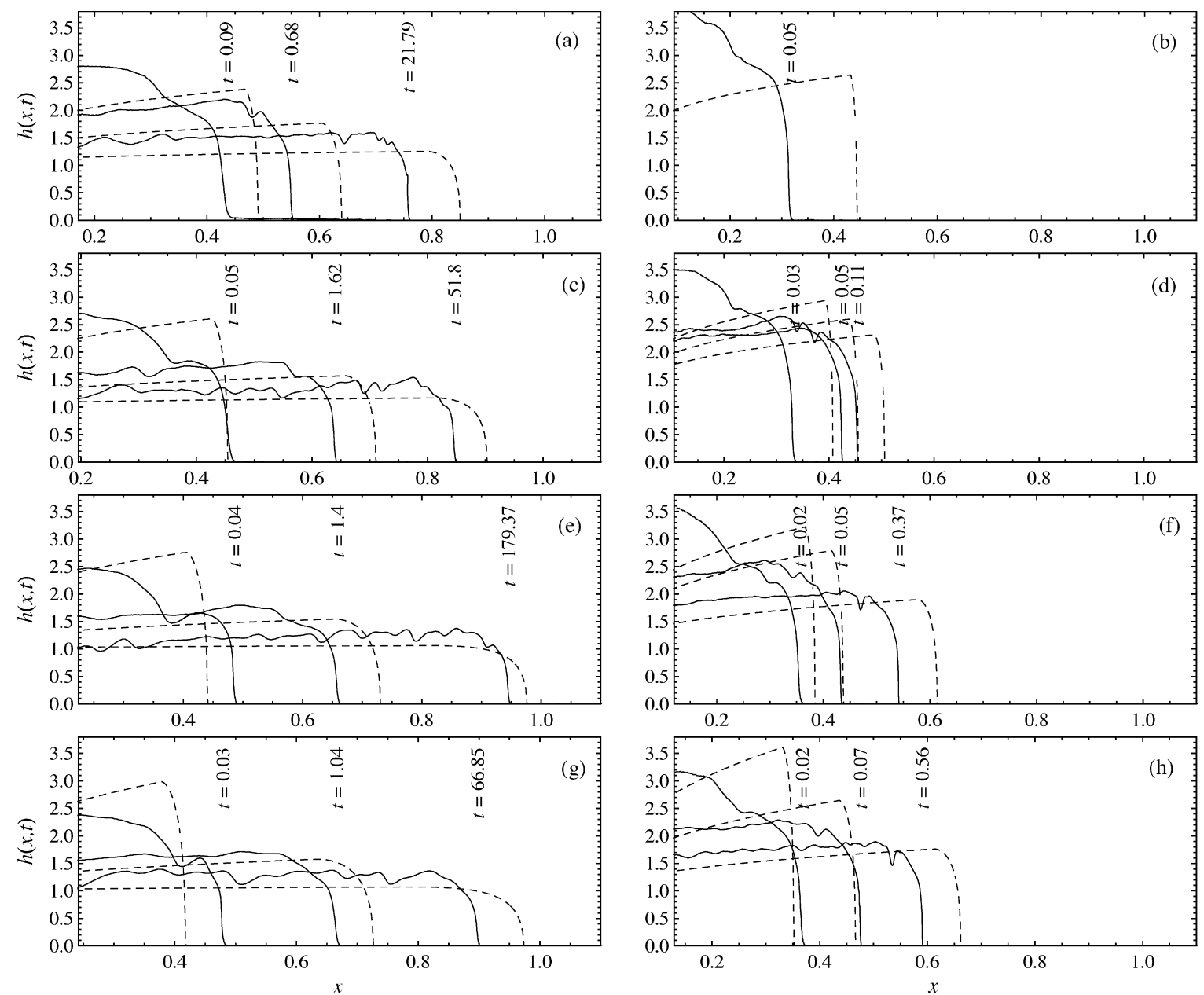

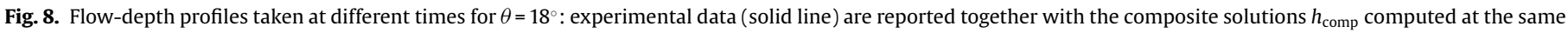
times (dashed line). The dimensionless time at which the profile is taken is also indicated just above the front; these times correspond to the dots plotted in Fig. 7.

condition for the evolution Eq. (53) is

$\left\{\begin{array}{l}h\left(x^{\prime}, 0\right)=h_{f} \text { for } x^{\prime} \leq 0, \\ h\left(x^{\prime}, 0\right)=0 \text { for } x^{\prime}>0\end{array}\right.$

The initial-boundary-value problem (53) and (54) must be solved numerically. For this purpose we used the pdepe routine provided in Matlab to solve parabolic differential equations in one space variable.

After substituting the stretched variables $\left(x^{\prime}, t^{\prime}\right)$ with the original scaled variables $\left(x=x_{\mathrm{f}}+\varepsilon x^{\prime}, t=\varepsilon t^{\prime}\right)$ in the solution to Eq. (53), we obtain a composite solution made up of the outer solution $h_{\text {outer }}$ and the inner solution $h_{\text {inner }}$

$h_{\text {comp. }}=h_{\text {outer }}+h_{\text {inner }}-h_{\text {front }}$,

where $h_{\text {front }}=h_{\mathrm{f}}$ is their overlap value, i.e., the flow depth at the front of the outer solution, $h_{\text {outer }}$ the solution to (45), and $h_{\text {inner }}$ the solution to (53). The composite solution provided a uniform approximation of the solution to leading order.

As shown in Fig. 3, the flow-depth profile is influenced a great deal by channel slope. At steep slopes (see Fig. 3(b)), the flow head is characterized by a blunt nose occupying a small fraction of the total length. In contrast, at shallow slopes (see Fig. 3(a)), the leading edge is acute and extends over most of the flow. This also shows that the position of the front is fairly accurately predicted by the outer solution at the steepest slopes, while we need to compute the full solution for the shallowest slopes.

\section{Experiments}

\subsection{Experimental facility}

We used a 30-cm-wide, 4-m-long flume fed by a reservoir, as sketched in Fig. 1. The flume laid on an aluminium plate, which was $4 \mathrm{~m}$ long, $1.8 \mathrm{~m}$ wide, and could be inclined from $0^{\circ}$ to $45^{\circ}$. Its position was accurately controlled using a digital inclinometer with a resolution of $0.1^{\circ}$.

The reservoir was positioned at the top of the inclined plane behind the dam wall. The maximum capacity of the reservoir was $120 \mathrm{~kg}$. The dam wall was composed of a $1.6 \mathrm{~m} \times 0.8 \mathrm{~m}$ ultralight carbon plate. Two pneumatic jacks opened the lock gate at the desired aperture within $0.5 \mathrm{~s}$. An ultralight dam wall was needed to reduce dam-wall inertia, plane vibration, and jerk. The two jacks were quickly raised by injecting air pressured at $7 \mathrm{MPa}$. Two electromagnetic sensors were located at the tip of each jack to control its position and reset the clock.

Before each run, the fluid was gently poured into the reservoir, while the inclined plane was kept in the horizontal position. The flume was then inclined at a given slope. The free surface was then carefully smoothed out until it was horizontal. At time $t=0$, the sluice gate was raised and the material started accelerating 

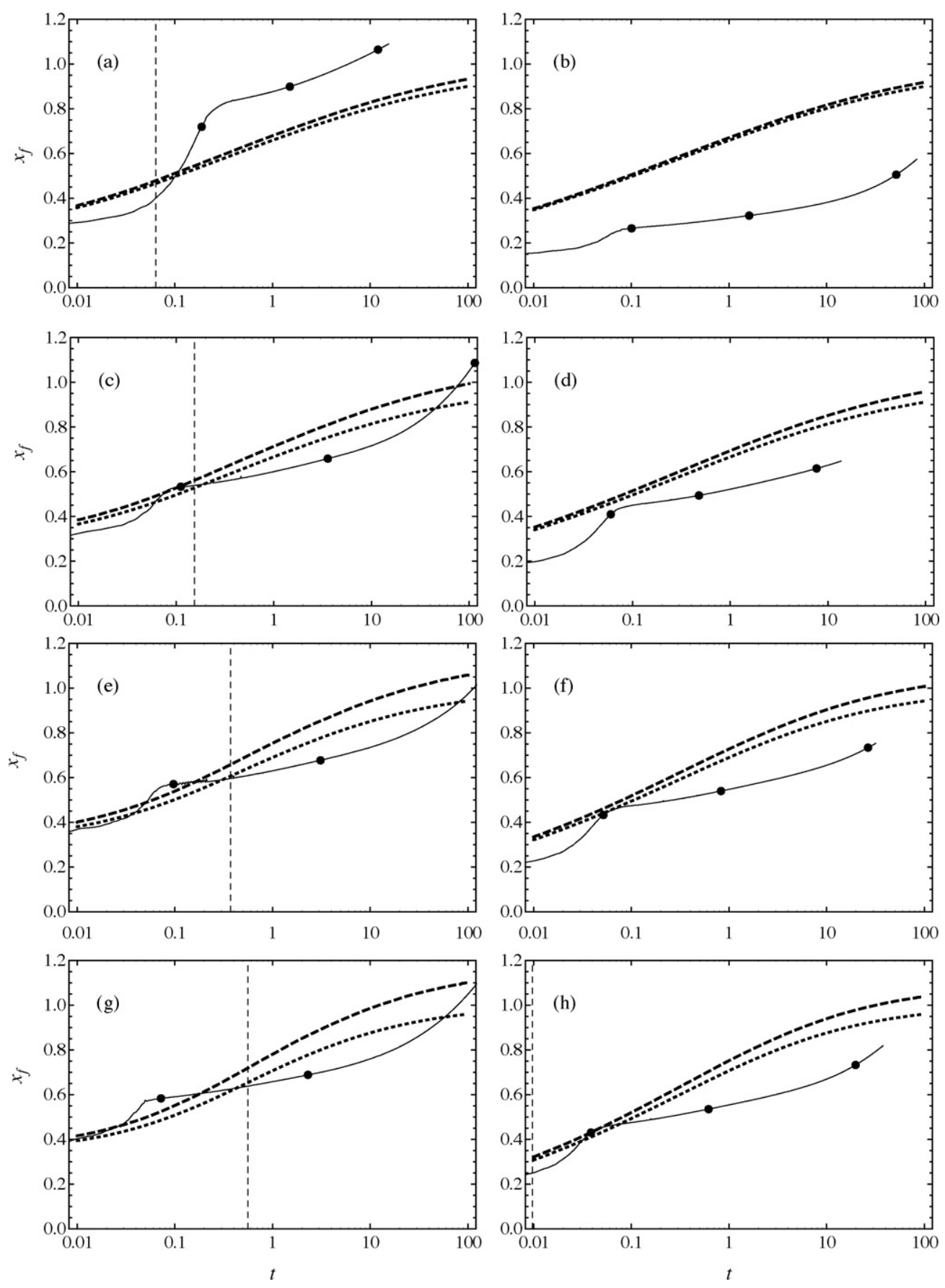

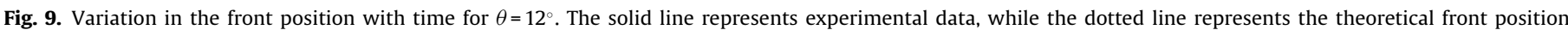

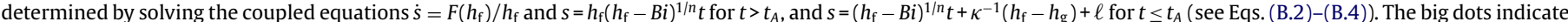

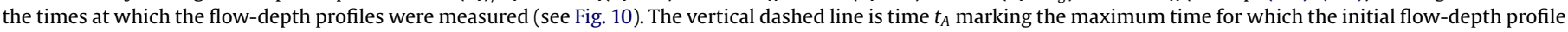
still influences the flow (see Appendix B). The dashed line stands for the position of the front for the composite solution $x_{\mathrm{f}}+\varepsilon x_{\mathrm{f}}^{\prime}($ see Section 2.3 ).

and flowing. The surge motion was imaged by a digital camera. When the front went beyond the imaged area, we stopped recording images. The material was then removed from the flume and the plane was carefully cleaned out.

To measure accurately the surge's free-surface variations with time, we have developed a new imaging system, consisting of a digital camera (Basler A202k Pixels camera provided by Qualimatest, Geneva, Switzerland) coupled with a synchronized micromirror projector (modified z-Snapper provided by ViaLux, Chemnitz, Germany). The object's surface was imaged into a camera and patterns were projected onto the surface under an angle of incidence that differed from the imaging direction $[44,45]$. From the deformed pattern recorded by the camera, the phase could be extracted and, using unwrapping algorithms, the height was computed and the free surface reconstructed. We were able to measure the free surface of the flow to within $1 \mathrm{~mm}$ every $22 \mathrm{~ms}$.

Fig. 4 shows a typical run, with both real and reconstructed free surfaces. We measured the flow-depth profile at the centerline of the flow. To attenuate noise effects, the flow depth was averaged over at 10-pixel band along the centerline (approx- 

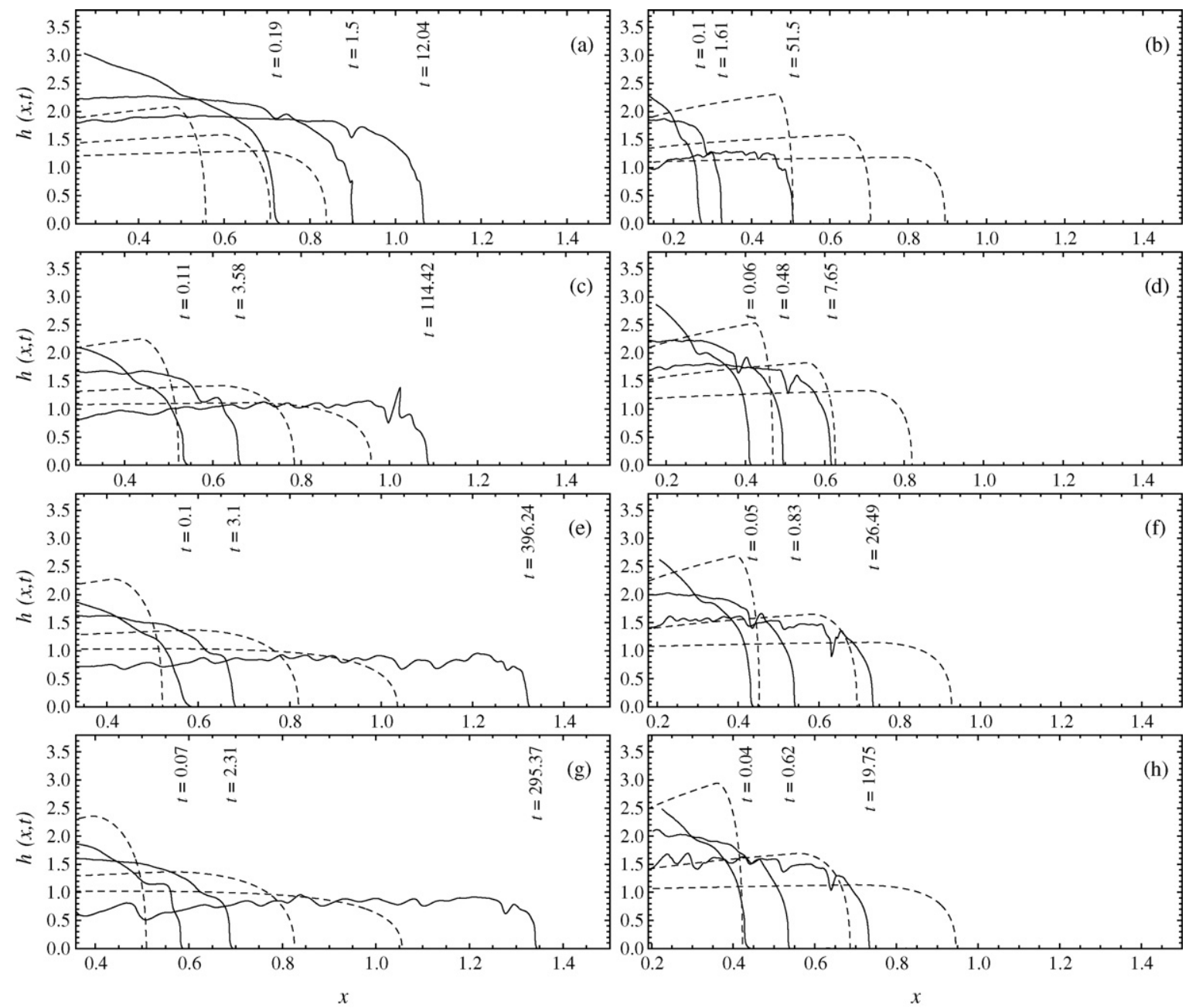

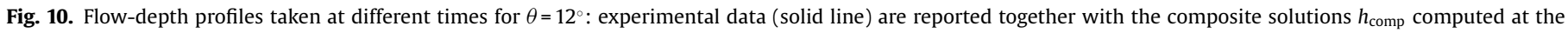
same times (dashed line). The dimensionless time at which the profile is taken is also indicated just above the front; these times correspond to the dots plotted in Fig. 9.

imately $1 \mathrm{~cm}$ ). The position of the front was evaluated at the flow centerline seeking the position at which the flow thickness dropped below a given threshold. On some occasions, locating the front accurately was difficult because of glints arising at the free surface near the contact line; these glints blurred the projected patterns and introduced noise in the post-treatment phase. The uncertainty on the front position could then be as high as $5 \mathrm{~mm}$.

\subsection{Material}

We used a viscoplastic stable polymeric gel called Carbopol Ultrez 10, produced by Noveon and provided by Gattefossé (Luzern, Switzerland). Anhydrous NaOH Pellets RPE-ACS-ISO (provided by Reactolab SA, Servion, Switzerland) were used to neutralize the Carbopol solution. The solvent was demineralized water. Carbopol Ultrez 10 is weakly thixotropic and viscoelastic like other Carbopol gels $[50,51]$. Over quite a wide range of shear rates, its rheological behavior can be closely approximated by a Herschel-Bulkley model. The rheological properties depend a great deal on the Carbopol concentration. Table 1 reports the Herschel-Bulkley parameters adjusted on our data as a function of the mass concentration in Carbopol. The density is $\rho=1000 \mathrm{~kg} \mathrm{~m}^{-3}$. See Ref. [52] for additional information.
The flow curve of the viscoplastic gel was determined using a Bohlin CVOR rheometer equipped with a Couette cell. We first determined the yield stress using a creep test [52]. We then determined the flow curve, i.e., the shear-stress/shear-rate relation. To that end, we used a standard technique, which involved imposing a step-like ramp of stress and recording the resulting deformation until equilibrium was reached (i.e., shear rate was constant). We determined the flow curve by solving the Couette inverse problem using Tikhonov regularization techniques [53]. We adjusted the Herschel-Bulkley simple-shear-flow equation

$\sigma_{x y}=\tau_{\mathrm{c}}+K \dot{\gamma}^{n}$

on the resulting data. $\tau_{\mathrm{c}}$ was set to the value determined by the creep test while $K$ and $n$ were computed using a least-square approach.

Table 1

Rheological characteristics of the Carbopol samples used

\begin{tabular}{lllll}
\hline Concentration & $0.25 \%$ & $0.30 \%$ & $0.35 \%$ & $0.40 \%$ \\
\hline$\tau_{\mathrm{c}}(\mathrm{Pa})$ & 78 & 89 & 102 & 109 \\
$K\left(\mathrm{~Pa} \mathrm{~s}^{-n}\right)$ & 32.1 & 47.68 & 58.91 & 75.84 \\
$n(-)$ & 0.388 & 0.415 & 0.505 & 0.579 \\
\hline
\end{tabular}



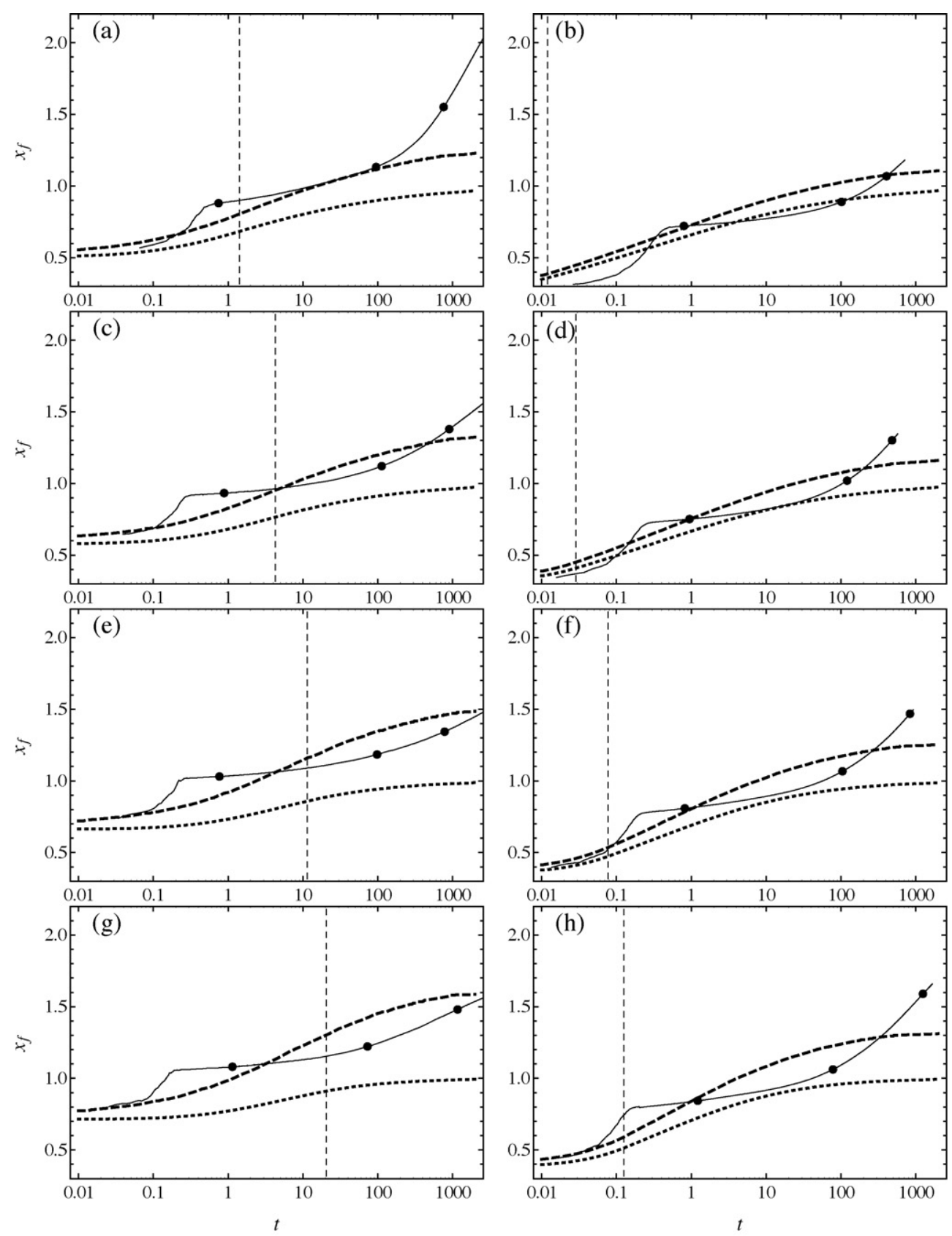

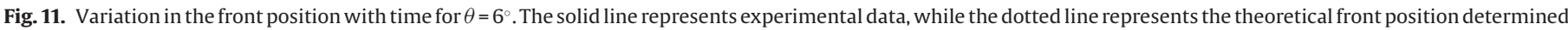

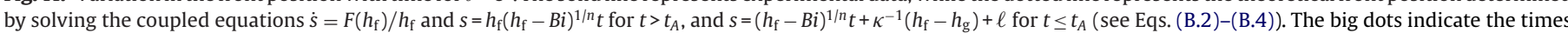

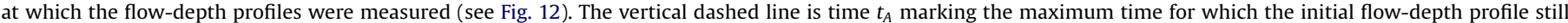
influences the flow (see Appendix B). The dashed line stands for the position of the front for the composite solution $x_{\mathrm{f}}+\varepsilon x_{\mathrm{f}}^{\prime}($ see Section 2.3 ).

\subsection{Experimental results for $\theta=24^{\circ}$}

Table 2 summarizes the main parameters for all runs carried out for $\theta=24^{\circ}$. We report two values for the aspect ratio $\varepsilon$ : its initial value $\varepsilon_{0}=h_{\mathrm{g}} / \ell$ and its final value $\varepsilon_{\mathrm{f}}=h_{\mathrm{c}} / \ell_{\mathrm{c}}=h_{\mathrm{c}}^{2} / V$ (when the material approaches the arrested state). With the latter scaling, the Bingham number is always unity; we also report the initial value taken by the Bingham number $B i_{0}$ computed with $\varepsilon=\varepsilon_{0}$ (that is, $H_{*}=h_{\mathrm{g}}$ and $L_{*}=\ell$ ). For all runs, the released mass was the same $(23 \mathrm{~kg})$ and sole the rheological parameters of the Carbopol samples varied.

Fig. 5 reports the variation in the front position with time. We have plotted both experimental data and theoretical curves given by the outer solution (dashed lines) provided in Appendix B and the composite solution (dotted lines) worked out in Section 2.3. The vertical dashed line marks the limit of influence of the initial flow depth $\left(t<t_{A}\right)$ for the dam-break problem (see Appendix B). On the whole, agreement is good between experiments and theory, the only significant difference being observed at early times during the slumping phase, when the front vigorously accelerated. There is also a slight lag between experimental and theoretical curves, which can be positive or negative. The general impression is that the computed front velocity is slightly higher than the observed one. On the same plot, the dots represent the times at which the flow-depth profiles reported in Fig. 6 were measured; three times were selected (short, intermediate, long times). Concerning the two 

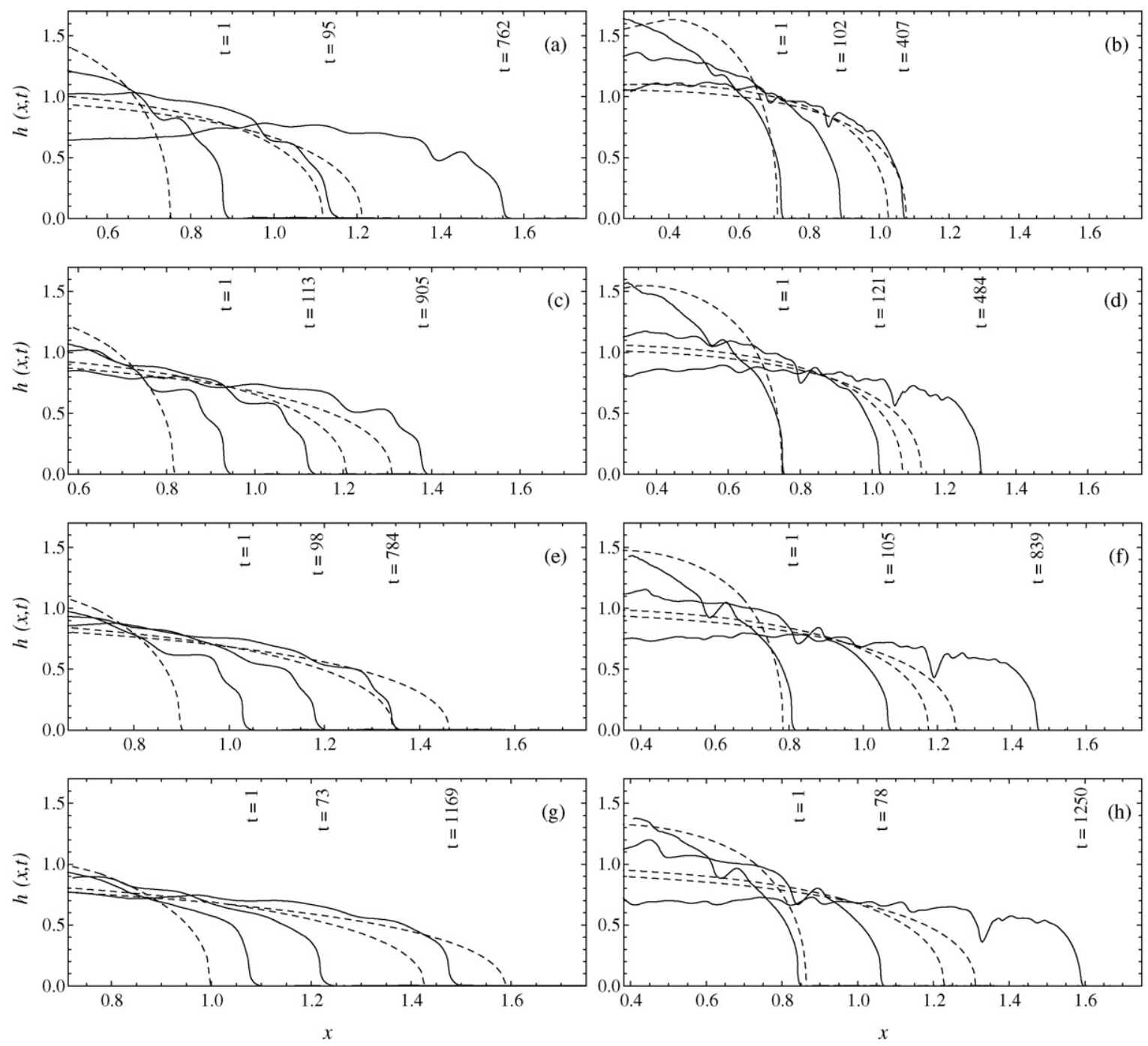

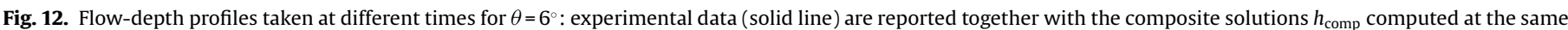
times (dashed line). The dimensionless time at which the profile is taken is also indicated just above the front; these times correspond to the dots plotted in Fig. 11.

theoretical approximations, note that (i) there is little difference between the outer and composite solutions and (ii) there is no change in behavior of the $x_{\mathrm{f}}(t)$ curves at the transition time $t=t_{A}$, which shows that here, the details of the initial flow-depth profile are of little importance to determining the behavior of the flow after the release. This is in line with Huang and García's findings [14].

In practice, because of the limited length of the imaged area, we could not reconstruct the free surface close to the reservoir, which implies that only measurements for $1.5<x \mid \ell<6$ $(0.2<x<0.9$ in a dimensionless form here) were taken. The main characteristics of the flow-depth profile (magnitude, over- all shape, front) are correctly described with the composite solution:

- On the whole, the shape of the avalanching mass is the same: a steep front is followed by a body with a nearly constant flow depth. In addition to the slight lag between experimental and theoretical curves.

- Naturally, the real flow-depth profiles are more irregular than the theoretical profiles: the more concentrated in Carbopol the sample was, the more corrugated the free surface was.

Table 2

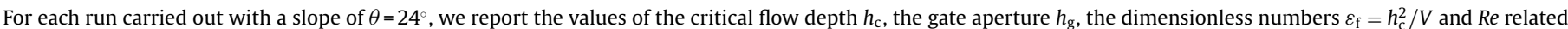
to the final (arrested) state, the initial value of the aspect ratio $\varepsilon_{0}=h_{g} / \ell$ and Bingham number $B i_{0}$

\begin{tabular}{|c|c|c|c|c|c|c|c|c|c|}
\hline Run & $h_{\mathrm{c}}(\mathrm{m})$ & $h_{\mathrm{g}}(\mathrm{m})$ & $\varepsilon_{\mathrm{f}}$ & $\varepsilon_{0}$ & $B i_{0}$ & $\operatorname{Re}$ & $n$ & $\left(\mathrm{~Pa} \mathrm{~s}^{-n}\right)$ & $\tau_{\mathrm{c}}(\mathrm{Pa})$ \\
\hline (a) & 0.019 & 0.26 & $4.9 \times 10^{-3}$ & 0.52 & 0.07 & 0.47 & 0.39 & 32.10 & 78.00 \\
\hline (b) & 0.022 & 0.26 & $6.4 \times 10^{-3}$ & 0.52 & 0.08 & 0.11 & 0.42 & 47.68 & 89.00 \\
\hline (c) & 0.025 & 0.26 & $8.5 \times 10^{-3}$ & 0.52 & 0.10 & 0.05 & 0.51 & 58.91 & 102.00 \\
\hline (d) & 0.027 & 0.26 & $9.9 \times 10^{-3}$ & 0.52 & 0.10 & 0.02 & 0.58 & 75.84 & 110.00 \\
\hline
\end{tabular}

The rheological parameters $n, K$, and $\tau_{c}$ are also recalled for convenience (see Table 1 ). The reservoir length was $\ell=0.51$. 
Table 3

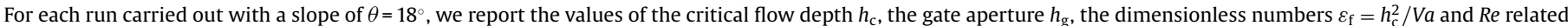
to the final (arrested) state, the initial value of the aspect ratio $\varepsilon_{0}=h_{\mathrm{g}} / \ell$ and Bingham number $B i_{0}$

\begin{tabular}{|c|c|c|c|c|c|c|c|c|c|}
\hline Run & $h_{\mathrm{c}}(\mathrm{m})$ & $h_{\mathrm{g}}(\mathrm{m})$ & $\varepsilon_{\mathrm{f}}$ & $\varepsilon_{0}$ & $B i_{0}$ & $R e$ & $n$ & $K\left(\mathrm{~Pa} \mathrm{~s}^{-n}\right)$ & $\tau_{\mathrm{c}}(\mathrm{Pa})$ \\
\hline (a) & 0.025 & 0.23 & $8.6 \times 10^{-3}$ & 0.46 & 0.11 & 0.82 & 0.39 & 32.10 & 78.00 \\
\hline (b) & 0.025 & 0.36 & $4.6 \times 10^{-3}$ & 0.71 & 0.07 & 0.82 & 0.39 & 32.10 & 78.00 \\
\hline (c) & 0.029 & 0.23 & $1.1 \times 10^{-3}$ & 0.46 & 0.13 & 0.19 & 0.42 & 47.68 & 89.00 \\
\hline (d) & 0.029 & 0.36 & $0.6 \times 10^{-3}$ & 0.71 & 0.08 & 0.19 & 0.42 & 47.68 & 89.00 \\
\hline (e) & 0.033 & 0.23 & $14 \times 10^{-3}$ & 0.46 & 0.14 & 0.09 & 0.51 & 58.91 & 102.00 \\
\hline (f) & 0.033 & 0.36 & $7.8 \times 10^{-3}$ & 0.71 & 0.09 & 0.09 & 0.51 & 58.91 & 102.00 \\
\hline (g) & 0.036 & 0.23 & $17 \times 10^{-3}$ & 0.46 & 0.16 & 0.04 & 0.58 & 75.84 & 110.00 \\
\hline (h) & 0.036 & 0.36 & $9.1 \times 10^{-3}$ & 0.71 & 0.10 & 0.04 & 0.58 & 75.84 & 110.00 \\
\hline
\end{tabular}

The rheological parameters $n, K$, and $t_{c}$ are also recalled for convenience (see Table 1 ). The reservoir length was $\ell=0.51$.

Table 4

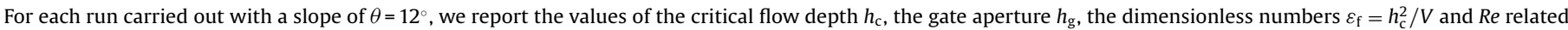
to the final (arrested) state, the initial value of the aspect ratio $\varepsilon_{0}=h_{\mathrm{g}} / \ell$ and Bingham number $B i_{0}$

\begin{tabular}{|c|c|c|c|c|c|c|c|c|c|}
\hline Run & $h_{\mathrm{c}}(\mathrm{m})$ & $h_{\mathrm{g}}(\mathrm{m})$ & $\varepsilon_{\mathrm{f}}$ & $\varepsilon_{0}$ & $B i_{0}$ & Re & $n$ & $K\left(\mathrm{~Pa} \mathrm{~s}^{-n}\right)$ & $\tau_{\mathrm{c}}(\mathrm{Pa})$ \\
\hline (a) & 0.038 & 0.20 & $1.9 \times 10^{-2}$ & 0.40 & 0.19 & 1.82 & 0.39 & 32.10 & 78.00 \\
\hline (b) & 0.038 & 0.34 & $1.0 \times 10^{-2}$ & 0.66 & 0.11 & 1.82 & 0.39 & 32.10 & 78.00 \\
\hline (c) & 0.043 & 0.20 & $2.4 \times 10^{-2}$ & 0.40 & 0.21 & 0.43 & 0.42 & 47.68 & 89.00 \\
\hline (d) & 0.043 & 0.34 & $1.3 \times 10^{-2}$ & 0.66 & 0.13 & 0.43 & 0.42 & 47.68 & 89.00 \\
\hline (e) & 0.050 & 0.20 & $3.2 \times 10^{-2}$ & 0.40 & 0.24 & 0.21 & 0.51 & 58.91 & 102.00 \\
\hline (f) & 0.050 & 0.34 & $1.7 \times 10^{-2}$ & 0.66 & 0.15 & 0.21 & 0.51 & 58.91 & 102.00 \\
\hline (g) & 0.053 & 0.20 & $3.7 \times 10^{-2}$ & 0.40 & 0.26 & 0.09 & 0.58 & 75.84 & 110.00 \\
\hline (h) & 0.053 & 0.34 & $2.0 \times 10^{-2}$ & 0.66 & 0.16 & 0.09 & 0.58 & 75.84 & 110.00 \\
\hline
\end{tabular}

The rheological parameters $n, K$, and $\tau_{\mathrm{c}}$ are also recalled for convenience (see Table 1 ). The reservoir length was $\ell=0.51$.

- The only significant difference is the early-time behavior, where the shape of the collapsing mass substantially differed from the one predicted by the composite solution, which is normal since our theoretical approximation does not hold for large-aspectratio flows, in particular if they are in an inertial phase as expected during the slumping phase.

\subsection{Experimental results for $\theta=18$}

We repeated our experimental procedure with the flume inclined at $\theta=18^{\circ}$ to the horizontal. We released either 23$\mathrm{kg}$ or $43-\mathrm{kg}$ masses of Carbopol (i.e., gate aperture $h_{\mathrm{g}}$ fixed at 26 or $36 \mathrm{~cm}$, respectively). We also changed the rheological properties of the fluid by altering the Carbopol concentration. All important parameters for each run are summarized in Table 3.

The same remarks as those made for $\theta=24^{\circ}$ hold here, in particular:

- The time variation in the front position $x_{\mathrm{f}}$ is fairly well predicted by theory, with a maximum deviation between theory and experiment of $15 \%$ (except for the early times $t<0.1$ ).
- There is no significant difference between the outer and composite solutions for the front position.

- Except for the slight lag time between theory and experiments, the predicted flow-depth profile $h_{\text {comp }}(x, t)$ is in good agreement with experimental data. In particular, the size of the head and the flow-depth gradient of the body are closely approximated by the composite solution.

Note that for the 43-kg mass (runs (b), (d), (f), and (h)), the front velocity was quite high compared to that reached by the 23$\mathrm{kg}$ mass, but not sufficiently high for a convective regime to be achieved. This explains why the bulk of the flow was in a slumping regime (notably for run (a)) and the experimental $x_{\mathrm{f}}$ deviated from the theoretical curve.

\subsection{Experimental results for $\theta=12$}

As for $\theta=18^{\circ}$ and $24^{\circ}$, we report the experimental conditions corresponding to runs (a)-(h) in Table 4 . Fig. 9 shows the variation in the front position with time, while Fig. 10 shows flow-depth profiles taken at different times. The experimental procedure was strictly identical to the one used for $\theta=18^{\circ}$; in particular, we used two

Table 5

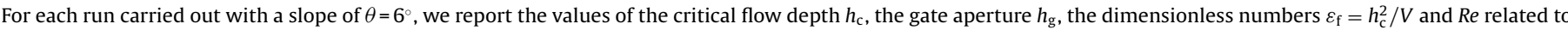
the final (arrested) state, the initial value of the aspect ratio $\varepsilon_{0}=h_{\mathrm{g}} / \ell$ and Bingham number $B i_{0}$

\begin{tabular}{|c|c|c|c|c|c|c|c|c|c|}
\hline Run & $h_{\mathrm{c}}(\mathrm{m})$ & $h_{\mathrm{g}}(\mathrm{m})$ & $\varepsilon_{\mathrm{f}}$ & $\varepsilon_{0}$ & $B i_{0}$ & $R e$ & $n$ & $K\left(\mathrm{~Pa} \mathrm{~s}^{-n}\right)$ & $\tau_{\mathrm{c}}(\mathrm{Pa})$ \\
\hline (a) & 0.076 & 0.177 & $7.5 \times 10^{-2}$ & 0.34 & 0.11 & 7.2 & 0.39 & 32.10 & 78.00 \\
\hline (b) & 0.076 & 0.307 & $7.5 \times 10^{-2}$ & 0.60 & 0.07 & 7.2 & 0.39 & 32.10 & 78.00 \\
\hline (c) & 0.086 & 0.177 & $3.9 \times 10^{-2}$ & 0.34 & 0.12 & 1.7 & 0.42 & 47.68 & 89.00 \\
\hline (d) & 0.086 & 0.307 & $3.9 \times 10^{-2}$ & 0.34 & 0.08 & 1.7 & 0.42 & 47.68 & 89.00 \\
\hline (e) & 0.099 & 0.177 & $2.9 \times 10^{-2}$ & 0.60 & 0.14 & 0.85 & 0.51 & 58.91 & 102.00 \\
\hline (f) & 0.099 & 0.307 & $2.9 \times 10^{-2}$ & 0.34 & 0.09 & 0.85 & 0.51 & 58.91 & 102.00 \\
\hline (g) & 0.10 & 0.177 & $2.0 \times 10^{-2}$ & 0.60 & 0.15 & 0.37 & 0.58 & 75.84 & 110.00 \\
\hline (h) & 0.10 & 0.307 & $2.0 \times 10^{-2}$ & 0.34 & 0.09 & 0.37 & 0.58 & 75.84 & 110.00 \\
\hline
\end{tabular}

The rheological parameters $n, K$, and $\tau_{c}$ are also recalled for convenience (see Table 1 ). The reservoir length was $\ell=0.51$. 
masses (23 and $43 \mathrm{~kg}$ ) and varied the rheological properties in the same way. To these two masses corresponded two gate apertures ( $h_{\mathrm{g}}=0.20 \mathrm{~m}$ and $0.34 \mathrm{~m}$, respectively) and initial $\varepsilon_{0}$ values $(\varepsilon=0.40$ and 0.66 , respectively).

Contrary to steep slopes, there is poor agreement between theory and experimental data concerning the front position. The theoretical curves not only deviate substantially from the experimental curves (up to $40 \%$ for run (b)), but also the shape is quite different: surprisingly enough, the experimental curves are convex, which shows that the front slightly accelerated
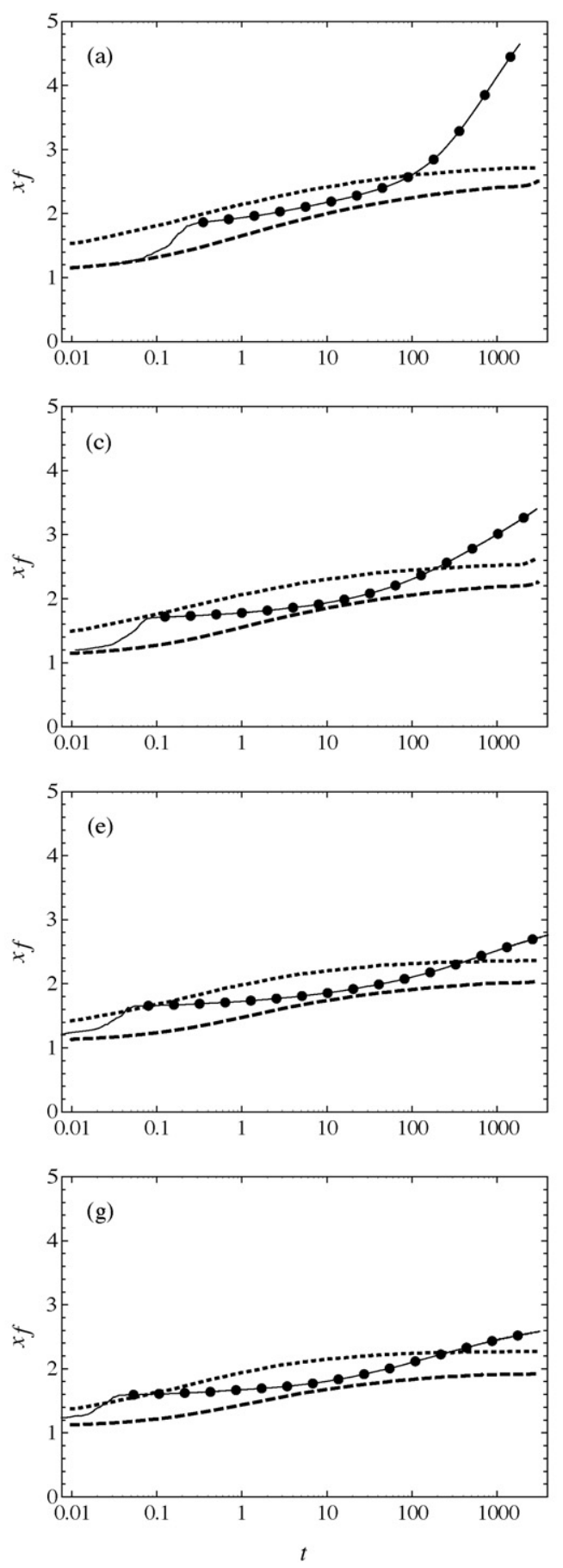

in spite of shallow slope, whereas the theoretical curves are grossly concave for small volumes, indicating front deceleration.

Agreement is somewhat better for the flow-depth profiles (except for early-time profiles), but taking a closer look at the body shape clearly shows that no nearly-uniform regime was achieved in this region for the 23-kg mass, whereas theory predicts that such a regime occurs at sufficiently long times (the flow depth is nearly constant for the dashed curves). For the large mass ( $43 \mathrm{~kg}$ ), this difference is not obvious.
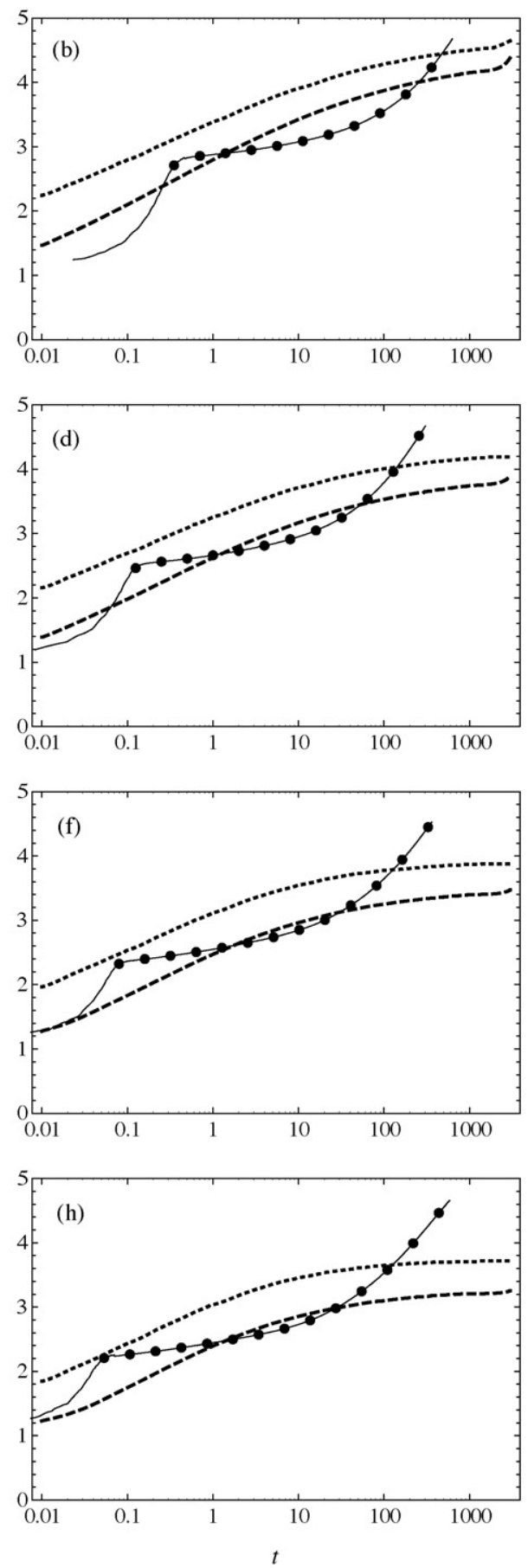

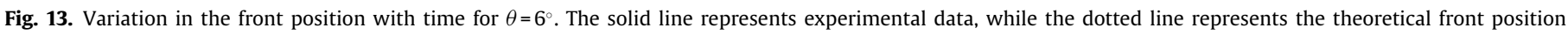

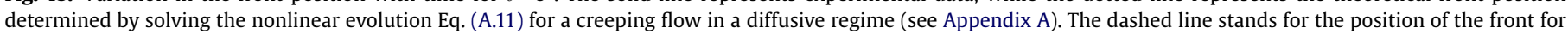
the composite solution $x_{\mathrm{f}}+\varepsilon x_{\mathrm{f}}^{\prime}$ (see Section 2.3) as for Fig. 11. For this figure we used a different scaling: $\varepsilon=\tan \theta(\operatorname{sothat} S=1), H_{*}=0.05 \mathrm{~m}\left(\right.$ arbitrary value), and $L *=H_{*} / \varepsilon$. 
At first glance, all these elements lead us to think that when slopes are mild, flows do not reach equilibrium; their dynamics seems to be controlled by gravitational forces, viscous dissipation, and pressure gradient, which makes it difficult any attempt to derive analytical approximation of the flow behavior. As we will see with experiments conducted at $6^{\circ}$, the bulk behavior is probably more complex than believed.

\subsection{Experimental results for $\theta=6^{\circ}$}

The experimental conditions are reported in Table 5. Fig. 11 shows the variation in the front position with time, while Fig. 12 shows flow-depth profiles taken at different times. The shortcomings pinpointed in Section 3.5 are exacerbated here. In particular, there are substantial differences between the theoretical and experimental $x_{\mathrm{f}}(t)$ curves. As noted in Section 3.5, the experimental curves are convex, which shows that the mass was slightly accelerating whereas theory predicts that the mass should have started decelerating and approaching the final state for $t \geq 100$. This surprising behavior cannot be easily understood unless we assume that the slight acceleration of the front is in fact due to a decrease in flow resistance or increase in supplied energy. The latter explanation can be discarded since the energy supplied by gravity acceleration remains constant, while elastic recovery is too low to affect the flow properties. The former explanation seems more plausible since a number of disturbing effects such as diffusion and bottom slip can affect the bulk behavior.

Since we are at shallow slopes, we can wonder whether the diffusive-regime theory outlined in Appendix A is more appropriate and yields better agreement with experiments. In Fig. 13, we reported the experimental $x_{\mathrm{f}}(t)$ curve together with the front position provided by the convective-regime theory $\left(x_{\mathrm{f}}+\varepsilon x_{\mathrm{f}}^{\prime}\right.$, dashed curve) and that given by the diffusive-regime theory (dotted curve). For the latter curve, we used the pdepe routine in Matlab to solve the nonlinear diffusion Eq. (A.11) numerically and determine the front position by seeking $x_{\mathrm{f}}$ such that $h\left(x_{\mathrm{f}}\right)=0$ (dotted curve). Sensitivity tests were also conducted on the initial time at which the mass was released. Indeed, since it took $0.5 \mathrm{~s}$ to open the lock gate (see Section 3.1) and part of the fluid was lifted up when removing the gate, the initial time was not known accurately. Numerical tests showed that the results were affected a great deal because of the vigorous acceleration experienced by the fluid during the slumping phase. This may somehow spoil comparison with experimental data because of this. This issue turned out to be of lower importance than initially believed since whatever the theoretical approximation used, we failed to reproduce the experimental curves: indeed they systematically exhibited convex shapes at sufficiently long times whereas the theoretical curves were concave and tended towards an asymptotic value $x_{\infty}$ (as shown in Appendix A). We then concluded that the discrepancies between theory and experiments could not be explained by the growing importance of diffusion effects at shallow slopes.

Wall slip can be another explanation for front acceleration. Indeed, slip is often associated with low-shear-stress regimes [54] and since at shallow slopes, the bottom shear stress was significantly lower than for steep slopes, slip could have occurred in spite of our efforts to remove or alleviate its effects. Careful examination of image records together with individual samples poured on the inclined did not provide any evidence that at shallow slopes, the head slipped along the bottom surface.

A third explanation was uncovered by looking at our movies, in particular movies recording unconfined flows down gentle slopes; a typical movie can be downloaded from our website http://lhe.epfl.ch/films/Carbopol.mpg. At the very beginning, after the material started flowing down the plane, the core of the flow

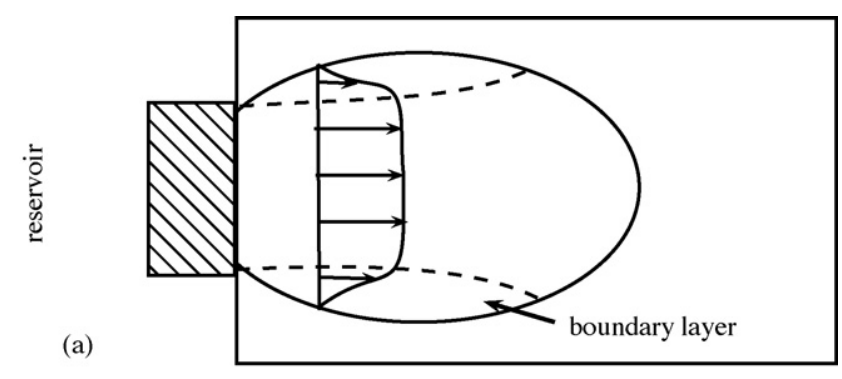

(b)

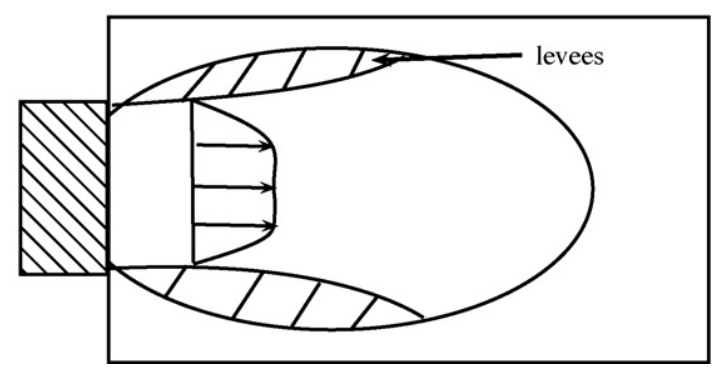

Fig. 14. Sketch depicting the sudden formation of lateral levees from weakly sheared rims for an unconfined flow: (a) once the flow was released, low-shear regions formed at the flow periphery. (b) Suddenly, the margins became unsheared and formed lateral levees confining the sheared material in the core region and the head.

was strongly sheared, whereas the fluid near the lateral rims experienced weak shear (see Fig. 14(a)). Once the flow width reached a nearly constant value, the rims 'froze' almost instantaneously and formed thick levees (see Fig. 14(b)). At the same time, a pulse originating from the flow rear overtook the front and gave new impetus to the head. This produced the kink that can be seen in all $x_{\mathrm{f}}(t)$ curves for unconfined flows [52] and to lesser extent for confined flows (in particular runs (b), (d), (f), and (h) in Fig. 12). Indeed, the flow rate remaining nearly constant over some period of time, flow narrowing caused by lateral levees led to swiftly increasing the mean velocity. If this scenario is correct, our two-dimensional analysis is too crude to capture the flow properties, notably the change in the front velocity induced by the levee formation. This scenario however remains speculative and calls for more work to elucidate this point.

\section{Conclusion}

In this paper, we investigated the behavior of a fixed volume of Herschel-Bulkley fluid down a sloping bed. With an appropriate scaling of the local governing equations and using matched asymptotic expansions, we derived an evolution equation for the flow depth, which takes on the form of a nonlinear convection equation for the body (outer solution). For the head (inner solution), a nonlinear diffusion equation is required to account for the large variations of the flow depth over short length scales. To leading order, the evolution equation for the flow depth within the body is similar to the equation worked out by Huang and García [14] using a kinematicwave approximation and Saint-Venant approach. For the head, the evolution equation differs: Huang and García [14] found that the flow-depth averaged velocity was uniform (independent of $x$ ) and equal to the front velocity within the tip region; this means that the leading edge behaves like a traveling wave. In our analysis, we found that the tip region was in a diffusive regime, which explains why the resulting governing equation looks like the nonlinear diffusion equation worked out by Liu and Mei [13] and Balmforth and Craster [38]. Compared to earlier work using the same framework as here (lubrication theory), the innovative point lies in the scaling, 
which makes it possible to derive an evolution equation for steep slopes, whereas with the scaling used in previous investigations, bed slope was necessarily shallow.

We also compared theory with experimental data. As Herschel-Bulkley fluids, we used Carbopol Ultrez 10, whose behavior in viscometric experiments can be closely approximated by the Herschel-Bulkley equation over a relatively wide range of shear rates. The rheological properties of our Carbopol samples were measured independently using a rheometer. Disturbing effects such as slip, thixotropy, and viscoelasticity, were negligible or controlled to a large extent. An experimental setup was designed to generate dam-break flows, i.e., a fixed volume of fluid was released onto an inclined flume. Using image processing techniques, we were able to accurately reconstruct the free surface of the avalanching mass at fairly high rates $(45 \mathrm{~Hz})$, which made it possible to track the free surface and contact line position over time. The flume inclination ranged from $6^{\circ}$ to $24^{\circ}$. The initial Bingham number $B i_{0}$ was in the 0.07-0.26 range, i.e., the samples fell into the low-yield-stress fluid category. $23-\mathrm{kg}$ and $43-\mathrm{kg}$ masses of Carbopol were tested, which led to different initial aspect-ratio values (initially, $\varepsilon_{0}$ was in the $0.3-0.7$ range).

Experiments at the highest slopes $\left(24^{\circ}\right)$ showed good agreement between theory and experimental data: both the front position and shape of the avalanching mass were correctly described by the zeroorder approximation of the governing equation. At milder slopes, discrepancies appeared and were exacerbated at gentle slopes. For shallow slopes, the substantial deviations between theory and experiments did not arise from diffusion effects or slip, but more probably from unsheared-zone (levee) formation, which made the flow structure three-dimensional. Note also that in agreement with theory, we did not observe a mass coming to a halt, which confirms that the final (arrested) state is not reached in finite time. This experimental observation contrasts with some observations made with kaolin (e.g., see the slump tests presented in Ref. [55]), where complete arrest was observed quickly after the release; this suggests that the choice of the material is essential to properly comparing theory and experiments for this kind of fluids.

\section{Acknowledgements}

The work presented here was supported by the Swiss National Science Foundation under grant number 200021-105193, the competence center in Mobile Information and Communication Systems (a center supported by the Swiss National Science Foundation under grant number 5005-67322, MICS project), the competence center in Environmental Sciences (TRAMM project), and specific funds provided by EPFL (vice-présidence à la recherche).

\section{Appendix A. Diffusive regime}

In this appendix, we outline the characteristics of the diffusive regime. With the dimensionless variables and scales introduced in Section 2, we end up with scaled governing equations:

$\varepsilon R e \frac{\mathrm{d} u}{\mathrm{~d} t}=S-\frac{\partial p}{\partial x}+\varepsilon \frac{\partial \sigma_{x x}}{\partial x}+\frac{\partial \sigma_{x y}}{\partial y}$,

$\varepsilon^{3} R e \frac{\mathrm{d} v}{\mathrm{~d} t}=-1+\frac{\partial p}{\partial y}+\varepsilon^{2} \frac{\partial \sigma_{x y}}{\partial x}+\varepsilon \frac{\partial \sigma_{y y}}{\partial y}$,

where $S=\tan \theta / \varepsilon$ is a slope parameter that can be set to unity unless the plane is horizontal. We pose the regular $\varepsilon$-expansions: $p=p_{0}+\varepsilon p_{1}+\ldots, h=h_{0}+\varepsilon h_{1}+\ldots$, and $\sigma=\sigma_{0}+\varepsilon \sigma_{1}+\ldots$, where $\sigma$ generically refers to the extra-stress components. If the Reynolds number is finite and we consider terms appearing to order $\varepsilon^{0}$, we have to solve

$0=S-\frac{\partial p_{0, x}}{\partial x}+\frac{\partial \sigma_{0, x y}}{\partial y}$

$0=-1-\frac{\partial p_{0}}{\partial y}$,

subject to $p_{0}=0$ and $\sigma_{0, x y}=0$ for $y=h_{0}$. On integrating these equations, we obtain

$\sigma_{0, x y}=\left(S-\partial_{x} h_{0}\right)\left(h_{0}-y\right)$

$p_{0}=h_{0}-y$,

We now pursue by inferring the velocity field $u$ from the stresses. By posing $u=u_{0}+\varepsilon u_{1}+\ldots$ and keeping zero-order terms, we derive

$\left(\frac{\partial u_{0}}{\partial y}\right)^{n}=\sigma_{0, x y}-B i$ for $\sigma_{0, x y}>B i$,

$\frac{\partial u_{0}}{\partial y}=0$ for $\sigma_{0, x y}<B i$,

The yield condition $\tau=B i$ is reached at elevation $y=Y_{0}(x, t)=h_{0}-B i\left|S-\partial_{x} h_{0}\right|^{-1}$. For, we obtain

$u_{0}(x, y, t)=\frac{n}{n+1}\left(S-\partial_{x} h_{0}\right)^{1 / n}\left(Y_{0}^{1+(1 / n)}-\left(Y_{0}-y\right)^{1+(1 / n)}\right)$,

while for $y \geq Y_{0}$, the velocity is constant to leading order: $u_{0}=$ $\frac{n}{n+1}\left(S-\partial_{x} h_{0}\right)^{1 / n} Y_{0}^{1+(1 / n)}$. Integrating the cross-stream velocity profile provides the flow-depth averaged velocity

$\bar{u}=\frac{n}{(n+2)(n+1)} Y_{0}\left[\left(S-\partial_{x} h_{0}\right) Y_{0}\right]^{1 / n} \frac{(2 n+1) h_{0}-n Y_{0}}{h_{0}}$.

Integrating the continuity equation provides the governing equation for $h$

$\frac{\partial h}{\partial t}+\frac{\partial}{\partial x} F(h)=0$,

with

$F(h)=n Y \frac{\left[\left(S-\partial_{x} h\right) Y\right]^{1 / n}((2 n+1) h-n Y)}{(n+2)(n+1)}$

and $Y=\max \left(h-B i\left|S-\partial_{x} h_{0}\right|^{-1}, 0\right)$.
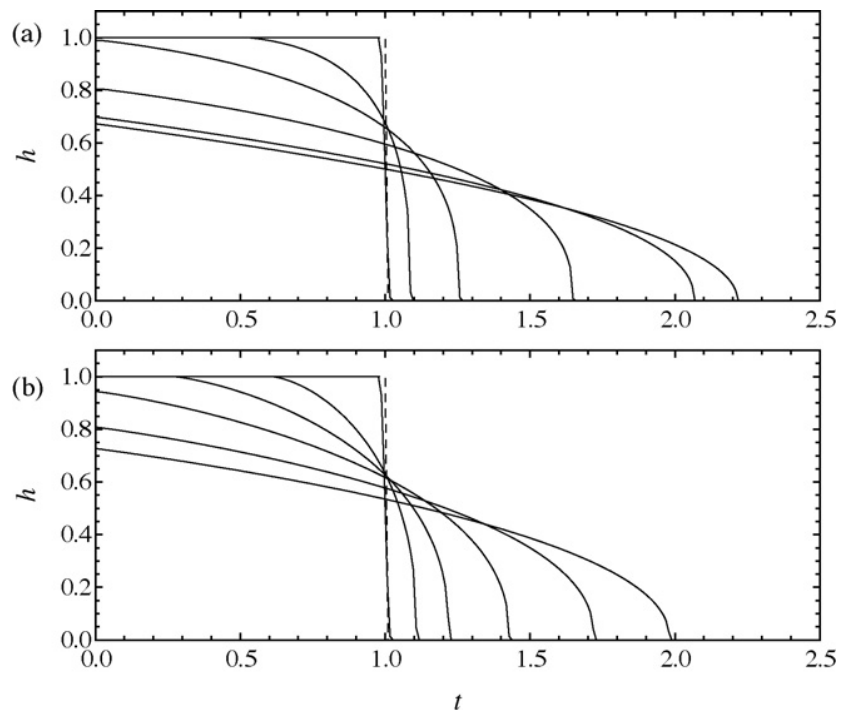

Fig. A1. (a) Flow-depth profiles for times $t=0.1,1,10$, and 1000 (solid line); the initial flow depth is also reported (dashed line). Numerical computations made for $\theta=0^{\circ}, \ell=1, B i=0.1, n=1, \Delta x=0.01, \Delta t=0.002$. (b) Flow-depth profiles with the same features as in (a) except that $n=1 / 2$. 
When $S>0$ and in the limiting case of the Newtonian ( $n=1$ and $B i=0)$ and power-law $(B i=0)$ fluids, this evolution equation does not admit similarity or other exact solutions and so must be integrated numerically; when $S=0$, similarity solutions can be worked out [41]. Fig. A1 shows the typical flow-depth profiles at different times, produced by the slump of a fixed volume on a dry horizontal boundary for two values of $n(n=1 / 2$ and $n=1)$. The flow-depth profile tends slowly towards a final stationary profile $h_{\infty}(x)$ for which $Y=0$. In the low-Bingham-number limit $(B i \leq 1 / 3)$, this profile is given by

$h_{\infty}(x)=\sqrt{2 B i\left(x_{\infty}-x\right)}$

with $x_{\infty}=(9 /(8 B i))^{1 / 3}$ the final position reached by the front [37,39]. Convergence is very slow, typically on the order of $t^{-n}$ [37]. The behavior and relevance of this equation to physical problems have been discussed in a number of earlier papers $[13,37,39]$ and therefore we will not pay more attention on it.

\section{Appendix B. Characteristic form}

To leading order, the governing equation for $h$ is given by Eq. (45), which can be recast in the characteristic form

$\frac{\partial h}{\partial r}=0$ along $\frac{\partial t}{\partial r}=1$ and $\frac{\partial x}{\partial r}=\partial_{h} F(h)=h(h-B i)^{1 / n}$,

subject to the constraint $h \geq B i$ and where $r$ is a dummy variable. This convection equation being hyperbolic, discontinuities may arise at $x=s(t)$ and propagate at a velocity $\dot{s}$ given by

$\dot{s} \llbracket h \rrbracket=\llbracket F(h) \rrbracket$

where $\llbracket h \rrbracket$, respectively $\llbracket F(h) \rrbracket$, is the jump experienced by $h$, respectively $F(h)$, across the shock located at $x=s(t)$.

It is straightforward to solve Eq. (B.1): using the initial conditions $t(0)=0, x(0)=x_{i}$, and $h(x, 0)=h_{i}\left(x_{i}\right)=h_{\mathrm{g}}+\kappa\left(x_{i}-\ell\right)$ given by $(25)$ and eliminating $r$, we find that the flow depth is the solution to the implicit equation

$h+\kappa h(h-B i)^{1 / n} t=h_{\mathrm{g}}+\kappa(x-\ell)$.

Let us only consider the case where the initial thickness $h_{i}$ is above $B i$ throughout the reservoir. In the converse case, this means that part of the volume in the reservoir will not flow once the lock gate is opened: for $h_{i}<B i$, i.e., for $0 \leq x \leq \kappa^{-1}\left(B i-h_{\mathrm{g}}\right)+\ell$, there will be no motion. This is in fact equivalent to considering a reservoir, the length of which is decreased by $\kappa^{-1}\left(B i-h_{\mathrm{g}}\right)+\ell$. In the following, we then assume that $B i<h_{\mathrm{g}}-\kappa \ell$.

Initially, at $x=0$ and $x=\ell$, the flow depth discontinuously drops to 0 , which gives rise to either a rarefaction wave or a shock $[56,57]$. On the right of the reservoir, the initial discontinuity at the lock gate necessarily causes the formation of a shock, which propagates rightward at the velocity $\dot{s}$ prescribed by (B.2): $\dot{s}=F\left(h_{\mathrm{f}}\right) / h_{\mathrm{f}}$, where $h_{\mathrm{f}}$ denotes the flow depth at the front and is evaluated using (B.3) at $x=s$. On the left, a centered rarefaction wave must occur and propagate from the rear end into the tail of the avalanching mass (see Fig. 5). Its features are deduced by seeking similarity solutions in the form $\mathcal{H}(\zeta)$ (with $\zeta=x / t$ ) to the convection Eq. (45) [56,58]. We find that $\mathscr{H}$ is implicitly given by

$\mathscr{H}(\zeta)(\mathscr{H}(\zeta)-B i)^{1 / n}=\zeta$

Except for some special values of $n$, this equation does not admit analytical solutions. For $n=1$ (Bingham fluid), we retrieve the similarity solution worked out by Huang and García [28]

$\mathscr{H}(\zeta)=\frac{1}{2}\left(B i+\sqrt{B i^{2}+4 \zeta}\right)$
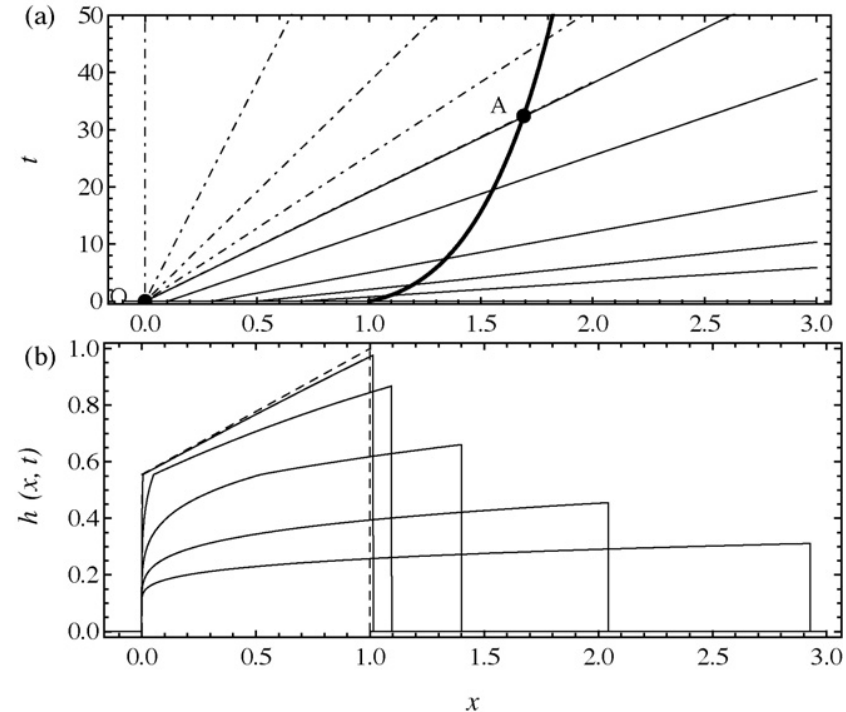

Fig. B1. (a) Characteristics of the convection Eq. (45) in the $x-t$ plane. The thin solid lines represent the characteristics emanating from $x=0$ with a slope imposed by the initial flow-depth profile $h_{i}\left(x_{i}\right)$. The dot-dashed lines are the characteristic fan originating from the point of origin $\mathrm{O}$ and representing the rarefaction wave at the tail of the avalanching mass. Their equation is $x=m t$ where $0 \leq m \leq m_{0}$ and $m_{0}=\left(h_{\mathrm{g}}-\kappa \ell\right)\left(h_{\mathrm{g}}-\kappa \ell-B i\right)^{1 / n}$. The thick line is the locus of the front position $x=s(t)$; at point $A$, the steepest characteristic $x=m_{0} t$ emanating from $O$ intersects the initial shock curve (representing the front motion at early times). (b) Evolution of the flowdepth profiles $h(x, y)$ with time. The profiles are computed at different times: $t=0$ (dashed line) and $t=10^{i}$ (solid lines), where $i=1,0, \ldots, 3$. Note that for $t<t_{A}=32.41$, there is a kink in the flow-depth profile, which stems from the initial discontinuity at the rear end. For $t<t_{A}=32.41$, the profiles are more regular and take a parabolic-like shape. Computations made for $\theta=24^{\circ}, \kappa=\tan \theta=0.44, \ell=1, B i=0.1, n=1 / 3$.

For $n=1 / 2$ (a reasonable approximation for many Herschel-Bulkley fluids), we find that

$\mathcal{H}(\zeta)=\frac{1}{6}\left(4 B i+\frac{2 \sqrt[3]{2} B i^{2}}{J(\zeta)}+2^{2 / 3} J(\zeta)\right)$

with $J(\zeta)=\sqrt[3]{-2 B i^{3}+27 \zeta+3 \sqrt{3} \sqrt{\zeta\left(27 \zeta-4 B i^{3}\right)}}$.

As shown in Fig. B1(a), the characteristics associated with this rarefaction wave form a fan of straight lines emanating from the point of origin $(x, t)=(0,0): x=m t$, with $m$ a parameter satisfying $0 \leq m \leq m_{0}$ and $m_{0}=\left(h_{\mathrm{g}}-\kappa \ell\right)\left(h_{\mathrm{g}}-\kappa \ell-B i\right)^{1 / n}$. At time $t_{A}$, the steepest characteristic coming from $O$ intersects the frontal shock curve $x=s(t)$ at point A. For time $t \leq t_{A}$, the flow-depth profile is piecewise continuous with $h(x, t)$ given by (B.3) for $m_{0} t \leq x \leq s(t)$ and by (B.4) for $0 \leq x \leq m_{0} t$. Time $t_{A}$ is the time at which the flow depth becomes independent of the initial conditions and conforms to a parabolic-like shape given by (B.4), as shown in Fig. B1(b). For $t \geq t_{A}$, the flow-depth profile is given by the similarity form (B.4). Fig. B1(b) shows flow-depth profiles taken at different times ranging from $t=0$ to $t=10^{3}$.

\section{References}

[1] A.M. Johnson, J.R. Rodine, Debris flow, in: D. Brunsden, D. Prior (Eds.), Slope Instability, John Wiley \& Sons, Chichester, 1984, pp. 257-362.

[2] Z. Wan, Z. Wang, Hypercontrated Flow, Balkema, Rotterdam, 1994.

[3] P. Coussot, Mudflow Rheology and Dynamics, Balkema, Rotterdam, 1997.

[4] J. Dent, T. Lang, Experiments on the mechanics of flowing snow, Cold Regions Sci. Technol. 5 (1982) 243-248.

[5] C. Ancey, Snow avalanches, in: N. Balmforth, A. Provenzale (Eds.), Geomorphological Fluid Mechanics: Selected Topics in Geological and Geomorphological Fluid Mechanics, Springer, Berlin, 2001, pp. 319-338.

[6] R.W. Griffiths, The dynamics of lava flows, Annu. Rev. Fluid Mech. 32 (2000) 477-518. 
[7] J.D. Dent, T.E. Lang, A biviscous modified Bingham model of snow avalanche motion, Ann. Glaciol. 4 (1983) 42-46.

[8] M. Kern, F. Tiefenbacher, J. McElwaine, The rheology of snow in large chute flows, Cold Regions Sci. Technol. 39 (2004) 181-192.

[9] R.M. Iverson, The physics of debris flows, Rev. Geophys. 35 (1997) 245296.

[10] R.M. Iverson, J. Vallance, New views of granular mass flows, Geology 29 (2002) 115-118.

[11] R.M. Iverson, The debris-flow rheology myth, in: C.L. Chen, D. Rickenmann (Eds.), Debris flow Mechanics and Mitigation Conference, Mills Press, Davos, 2003, pp. 303-314.

[12] C. Ancey, Plasticity and geophysical flows: a review, J. Non-Newtonian Fluid Mech. 142 (2007) 4-35.

[13] K.F. Liu, C.C. Mei, Slow spreading of a sheet of Bingham fluid on an inclined plane, J. Fluid Mech. 207 (1990) 505-529.

[14] X. Huang, M.H. Garcìa, A Herschel-Bulkley model for mud flow down a slope, J. Fluid Mech. 374 (1998) 305-333.

[15] H. Chanson, S. Jarny, P. Coussot, Dam break wave of thixotropic fluid, J. Hydraul. Eng. 132 (2006) 280-293.

[16] K. Debiane, J.-M. Piau, Ecoulement généré par le lâcher instantané d'un barrage retenant un fluide viscoplastique, Les cahiers de rhéologie 18 (2001) 45-54.

[17] J.J. Major, Experimental studies of deposition of debris flows: process, characteristics of deposits, and effects of pore-fluid pressure, Ph.D., Washington, 1996.

[18] J.D. Parsons, K.X. Whipple, A. Simoni, Experimental study of the grain-flow, fluid-mud transition in debris flows, J. Geol. 109 (2001) 427-447.

[19] B. McArdell, P. Bartelt, J. Kowalski, Field observations of basal forces and fluid pore pressure in a debris flow, Geophys. Res. Lett. 34 (2006) L07406.

[20] M. Hürlimann, D. Rickenmann, C. Graf, Field and monitoring data of debris-flow events in the Swiss Alps, Can. Geotech. J. 40 (2003) 161-175.

[21] M. Berti, R. Genevois, A. Simoni, P. Tecca, Field observations of a debris flow event in the Dolomites, Geomorphology 29 (1999) 265-274.

[22] B. McArdell, B. Zanuttigh, A. Lamberti, D. Rickenmann, Systematic comparison of debris flow laws at the Illgraben torrent, Switzerland, in: C. Chen (Ed.), Debris flow Mechanics and Mitigation Conference, Mills Press, Davos, 2003, pp 647-657.

[23] P. Coussot, S. Proust, C. Ancey, Rheological interpretation of deposits of yield stress fluids, J. Non-Newtonian Fluid Mech. 66 (1996) 55-70.

[24] N.J. Balmforth, A.S. Burbridge, R.V. Craster, Viscoplastic models of isotherma lava domes, J. Fluid Mech. 403 (2000) 37-65.

[25] S. Baloga, L. Glaze, J. Crisp, S. Stockman, New statistics for estimating the bulk rheology of active lava flows: Puu Oo examples, J. Geophys. Res. 103 (B3) (1998) 5133-5142.

[26] H. Pinkerton, R.S.J. Sparks, Field measurements of the rheology of lava, Nature 276 (1978) 383-384

[27] D.I. Osmond, R.W. Griffiths, The static shape of yield strength fluids slowly emplaced on slopes, J. Geophys. Res. B 106 (2001) 16241-16250.

[28] X. Huang, M.H. Garcìa, A perturbation solution for Bingham-plastic mudflows, J. Hydraulic Eng. 123 (1997) 986-994.

[29] A. Siviglia, A. Cantelli, Effect of bottom curvature on mudflow dynamics: theory and experiments, Water Resour. Res. 41 (2005) W11423.

[30] J.-M. Piau, Consistometry slump and spreading tests: practical comments, J. Non-Newtonian Fluid Mech. 135 (2006) 177-178.

[31] J.-M. Piau, Flow of a yield stress fluid in a long domain. Application to flow on an inclined plane, J. Rheol. 40 (1996) 711-723.

[32] M. Pastor, M. Quecedo, E. González, M. Herreros, J. Fernández, P. Mira, Simple approximation to bottom friction for Bingham fluid depth integrated models, J. Hydraul. Eng. 130 (2004) 149-155.
[33] C.C. Mei, M. Yuhi, Slow flow of a Bingham fluid in a shallow channel of finite width, J. Fluid Mech. 431 (2001) 135-159.

[34] C.C. Mei, K.F. Liu, M. Yuhi, Mud flows - slow and fast, in: N. Balmforth, A. Provenzale (Eds.), Geomorphological Fluid Mechanics: Selected Topics in Geological and Geomorphological Fluid Mechanics, Springer, Berlin, 2001, pp. 548-577.

[35] K.F. Liu, C.C. Mei, Approximate equations for the slow spreading of a thin sheet of Bingham plastic fluid, Phys. Fluids A 2 (1990) 30-36.

[36] M. Yuhi, C.C. Mei, Slow spreading of fluid mud over a conical surface, J. Fluid Mech. 519 (2004) 337-358.

[37] G.P. Matson, A.J. Hogg, Two-dimensional dam break flows of Herschel-Bulkley fluids: the approach to the arrested state, J. Non-Newtonian Fluid Mech. 142 (2007) 79-94.

[38] N.J. Balmforth, R.V. Craster, A consistent thin-layer theory for Bingham plastics, J. Non-Newtonian Fluid Mech. 84 (1999) 65-81.

[39] N.J. Balmforth, R.V. Craster, P. Perona, A.C. Rust, R. Sassi, Viscoplastic dam breaks and the Bostwick consistometer, J. Non-Newtonian Fluid Mech. 142 (2007) 63-78.

[40] N.J. Balmforth, R.V. Craster, A.C. Rust, R. Sassi, Viscoplastic flow over an inclined surface, J. Non-Newtonian Fluid Mech. 142 (2007) 219-243.

[41] N.J. Balmforth, R.V. Craster, R. Sassi, Shallow viscoplastic flow on an inclined plane, J. Fluid Mech. 470 (2002) 1-29.

[42] J.R. Lister, Viscous flows down an inclined plane from point and line sources, J. Fluid Mech. 242 (1992) 631-653.

[43] P. Møller, J. Mewis, D. Bonn, Yield stress and thixotropy: on the difficulty of measuring yield stresses in practice, Soft Matter 2 (2006) 274-283.

[44] S. Cochard, Measurements of time-dependent free-surface viscoplastic flows down steep slopes, Ph.D. thesis, Ecole Polytechnique Fédérale de Lausanne, 2007.

[45] S. Cochard, C. Ancey, Tracking the free surface of time-dependent flows: image processing for the dam-break problem, Exp. Fluids 44 (2008) 59-71.

[46] J. Kevorkian, J.D. Cole, Multiple Scale and Singular Perturbation Methods, Springer, New York, 1996.

[47] R.B. Bird, G.C. Dai, B.J. Yarusso, The rheology and flow of viscoplastic materials, Rev. Chem. Eng. 1 (1983) 1-70.

[48] B. Hunt, Dam-break solution, J. Hydraul. Eng. 110 (1984) 675-686.

[49] B. Hunt, Newtonian fluid mechanics treatment of debris flows and avalanches, J. Hydraul. Eng. 120 (1994) 1350-1363.

[50] G.P. Roberts, H.A. Barnes, New measurements of the flow-curves for Carbopol dispersions without slip artefacts, Rheol. Acta 40 (2001) 499-503.

[51] S.J. Curran, R.E. Hayes, A. Afacan, M.C. williams, P.A. Tanguy, Properties of Carbopol solutions as models for yield-stress fluids, J. Food Sci. 67 (2002) $176-180$.

[52] S. Cochard, C. Ancey, Experimental investigation into the spreading of viscoplastic fluids onto inclined planes, J. Non-Newtonian Fluid Mech. 158 (2009) 73-84.

[53] C. Ancey, Solving the Couette inverse problem by using a wavelet-vaguelette decomposition, J. Rheol. 49 (2005) 441-460.

[54] H.A. Barnes, A review of the slip (wall depletion) of polymer solutions, emulsions and particle suspensions in viscometers: its cause, character, and cure, J. Non-Newtonian Fluid Mech. 56 (1995) 221-251.

[55] C. Ancey, H. Jorrot, Yield stress for particle suspensions within a clay dispersion, J. Rheol. 45 (2001) 297-319.

[56] R. Courant, K.O. Friedrich, Supersonic Flow and Shock Waves, Intersciences Publishers, New York, 1948.

[57] R.J. LeVeque, Finite Volume Methods for Hyperbolic Problems, Cambridge University Press, Cambridge, 2002.

[58] E. Zauderer, Partial Differential Equations of Applied Mathematics, Pure and Applied Mathematics, John Wiley \& Sons, New York, 1983. 